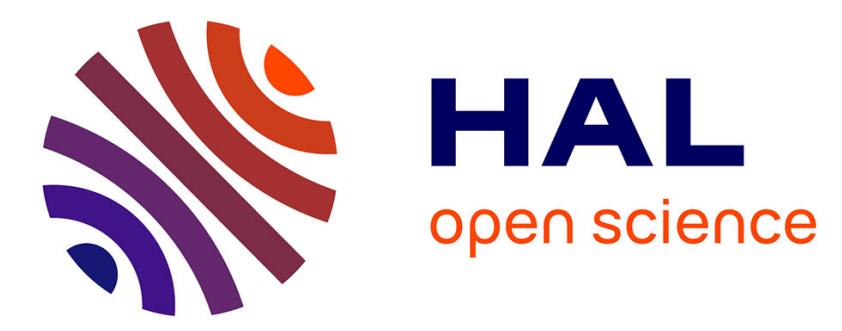

\title{
Nombrils, bruslans, autrement foyerz: la géométrie projective en action dans le Brouillon Project de Girard Desargues
}

Marie Anglade, Jean-Yves Briend

\section{- To cite this version:}

Marie Anglade, Jean-Yves Briend. Nombrils, bruslans, autrement foyerz: la géométrie projective en action dans le Brouillon Project de Girard Desargues. Archive for History of Exact Sciences, 2021, 10.1007/s00407-021-00282-3 . hal-02934376v3

\section{HAL Id: hal-02934376 \\ https: / hal-amu.archives-ouvertes.fr/hal-02934376v3}

Submitted on 24 Jul 2021

HAL is a multi-disciplinary open access archive for the deposit and dissemination of scientific research documents, whether they are published or not. The documents may come from teaching and research institutions in France or abroad, or from public or private research centers.
L'archive ouverte pluridisciplinaire HAL, est destinée au dépôt et à la diffusion de documents scientifiques de niveau recherche, publiés ou non, émanant des établissements d'enseignement et de recherche français ou étrangers, des laboratoires publics ou privés. 


\title{
Nombrils, bruslans, autrement foyerz : la géométrie projective en action dans le Brouillon Project de Girard Desargues
}

\author{
par Marie Anglade et Jean-Yves Briend \\ 2 I juillet 202 I
}

\begin{abstract}
Mais c'est mon plus beau temps! Le bonheur, comme on dit, n'habite pas sous des nombrils dorés.
\end{abstract}

Honoré de Balzac, Un début dans la vie.

\begin{abstract}
Résumé
Le Brouillon Project de Girard Desargues sur les coniques développe, dans sa partie centrale, la notion de traversale, qui généralise celle de diamètre d'Apollonius et permet d'unifier le traitement des diverses espèces de coniques. Nous avons montré ailleurs que le Lyonnais y développe une théorie complète de la polarité projective des coniques. Nous allons dans le présent article analyser la fin du Brouillon Project, où la théorie de la traversale est mise en pratique pour donner un très élégant traitement du paramètre et des foyers. Cela nous permettra de montrer que les preuves de Desargues ne peuvent se comprendre que si l'on accepte qu'il raisonne dans un cadre que l'on peut qualifier de projectif, assimilant complètement les éléments à l'infini à ceux à distance finie dans ses démonstrations.
\end{abstract}

\begin{abstract}
In the middle part of his Bronillon Project on conics, Girard Desargues develops the theory of the traversale, a notion that generalizes the Apollonian diameter and allows to give a unified treatment of the three kinds of conics. We showed elsewhere that it leads Desargues to a complete theory of projective polarity for conics. The present article, which shall close our study of the Brouillon Project, is devoted to the last part of the text, in which Desargues puts his theory of the traversal into practice by giving a very elegant tratment of the classical theory of parameters and foci. This will lead us to show that Desargues' proofs can only be understood if one accepts that he reasons in a resolutely projective framework, completely assimilating elements at infinity to those at finite distance in his proofs.
\end{abstract}




\section{Introduction}

Girard Desargues a été qualifié par Jean-Victor Poncelet de père de la géométrie projective. La lecture de son Bromillon Project sur les coniques de 1639, en particulier ses premières pages où sont confondues les notions de parallélisme et de concourance, semble confirmer la justesse de ce jugement. On peut cependant lui objecter le fait que les méthodes de démonstration de Desargues, s'appuyant sur la théorie des proportions d'Euclide et les théorèmes de Thalès ou de Ménélaüs, ne semblent en aucun cas être de nature projective. Nous avons montré dans nos articles [I] et [2] que progressivement, le Lyonnais abandonnait les réticences qu'il pouvait avoir au début du Bronillon à raisonner, sans les différencier, sur les points à distance finie ou infinie. Cela est illustré de manière spectaculaire par son introduction de la «droite à l'infinie » qui est vue comme la traversale (ou polaire, en termes modernes) du centre d'une conique eu égard à celle-ci, ce qui l'amène à écrire ${ }^{\mathrm{I}}:$ « Par forme d'éclaircissemens, Quand en un plan, aucun des poincts d'une droicte n'y est à distance finie, cette droicte y est à distance infinie. D'autant qu'en un plan le poinct nommé centre d'une coupe de rouleau n'est qu'un cas d'entre les innombrables buts d'ordonnances de droictes, il ne doit estre icy iamais parlé de centre de coupe de rouleau. » Ce qui est important ici c'est que l'objet considéré, bien que tous ses points soient à distance infinie, est bien une «droicte ».

Nous voudrions montrer dans cet article pourquoi l'on peut considérer que Desargues, dans le dernier tiers de son Browillon Project, raisonne et démontre d'une manière que l'on qualifiera plus tard de projective. Nous allons pour cela analyser de manière précise cette partie du texte, où Desargues met en application sa théorie projective de la polarité pour retrouver certaines notions et résultats classiques de la théorie apollonienne des coniques comme le paramètre ou le foyer.

Suite à la redécouverte des mathématiques antiques aux XVe et XVIe siècle en Europe est apparu le problème de comprendre, de simplifier, de restituer et d'étendre l'un des plus difficiles traités de l'antiquité grecque, Les Coniques d'Apollonius de Perge. Cela a motivé de nombreux travaux au début du XVIIe siècle et l'on peut dire que Descartes comme Desargues vont y apporter des contributions très importantes en introduisant deux révolutions dans la manière d'envisager la pratique de la géométrie : en la réduisant au calcul chez Descartes, en fondant la géométrie projective chez Desargues ${ }^{2}$. Il va sans dire qu'aux yeux de leurs contemporains, la mise à l'épreuve de ces nouveautés doit passer par une confrontation avec les propositions centrales du traité d'Apollonius. C'est à cela que sont consacrées les dernières pages ${ }^{3}$ du Bronillon, que nous allons ici analyser. La rédaction de Desargues y est souvent confuse et d'un style obscur. Il s'avère cependant qu'une fois que l'on accepte que Desargues raisonne de manière projective, certains passages qui jusque là étaient restés incompris ou savamment ignorés, même des meilleurs spécialistes du sujet, prennent sens et nous montrent un mathématicien qui pratique la géométrie comme personne ne le fera plus après lui pendant presque deux siècles.

I. Advertissement pour la ligne 3 de la page 16 .

2. Nous renvoyons au chapitre Style cartésien, style arguésien du livre [ı] de Gilles-Gaston Granger pour une analyse moins caricaturale.

3. Plus précisément les pages 23 à 29 de l'original, voir plus bas. 
Nous commencerons cet article par un bref rappel du contenu des vingt premières pages du texte, en insistant sur les notions d'arbre, d'involution et de traversale et en rappelant les quelques résultats importants que Desargues y démontre. Nous décrirons ensuite un court passage où le Lyonnais semble initier une théorie des couples de cercles ou de coniques, ce qui semble sans rapport avec la théorie apollonienne à laquelle il s'attaque juste après mais qui, comme nous le verrons par la suite, a toute sa place ici. Nous analyserons ensuite la brève et élégante théorie du paramètre développée dans le Bronillon, qui procède par spécialisations successives, montrant comment cette notion s'insère dans la théorie projective des coniques. Les foyers sont ensuite étudiés par Desargues d'une manière originale, en les raccrochant à sa théorie du paramètre et en les introduisant par une réciproque de la proposition 45 du livre III des Coniques d'Apollonius, proposition que le Lyonnais qualifie d'évidente et dont découlent facilement tous les résultats classiques les concernant.

C'est dans la section suivante, où nous analysons comment Desargues traite de l'hyperbole, qu'apparaîtront de manière éclatante les manières projectives de Desargues. Nous y verrons entre autre qu'il n'hésite pas à considérer le birapport de points situés sur la droite à l'infini. Avant de conclure le Brouillon par des considérations générales que nous n'aborderons pas ici, le Lyonnais donne une application de ses méthodes à une très belle construction de nature perspectiviste qui fait écho à la dernière phrase de son petit petit opuscule de 1636 sur la perspective ${ }^{4}$ : étant donnée une conique coupée par une droite et un point sur cette droite, il construit une perspective vers un autre plan de sorte que la figure devienne celle d'une conique, de son grand axe et d'un de ses foyers.

Cet article est le dernier d'une série de quatre (voir [I], [2] et [3] pour les trois premiers) que les auteurs ont consacrés à une analyse aussi complète qu'il se peut du contenu mathématique du Brouillon Project. Ce travail devrait déboucher sur une réédition commentée du traité du Lyonnais qui permette au plus grand nombre d'accéder aux idées et méthodes extraodinaires que l'on y trouve et de lever certains malentendus qui pèsent toujours sur ce texte.

\section{Introduction : english version}

Girard Desargues has been called by Jean-Victor Poncelet the father of projective geometry. The reading of his Browillon Project on conics of 1639, in particular its first pages where the notions of parallelism and concourance are unified, seems to confirm the accuracy of this judgement. However, one may object to the fact that Desargues' methods of proof, based on Euclid's theory of proportions and the theorems of Thales or Menelaos, do not seem to be projective in nature. We have shown in our articles [I] and [2] that gradually, the Lyonnais abandoned the reticence he might have had at the beginning of the Draft to reason, without differentiating, about points at finite or infinite distance. This is dramatically illustrated by his introduction of the "line at infinity" which is seen as the traversale (or polar, in modern terms) of the centre of a

4. Intitulé L'exemple de l'une des manières $d u S^{r}$. G. D. L. touchant à la pratique de la perspective, Sans employer aucun tiers poinct de distance ou d'autre nature qui soit hors du champs de l'Ouvrage. Ouvrage disponible sur le site GALLICA. 
conic, leading him to writes : "By way of clarification, When in a plane, none of the points of a line are at a finite distance, this line is at an infinite distance. Inasmuch as in a plane the point called the centre of a roll cut is only one of the innumerable butts of ordinances of lines, it must never be spoken here of the centre of a roll cut." What is important here is that the object considered, although all its points are at infinite distance, is indeed a line.

In this article we would like to show why we can consider that Desargues, in the last third of his Draft Project, reasons and demonstrates in a way that we will later qualify as "projective". To do this, we will analyse precisely this part of the text, where Desargues applies his projective theory of polarity to recover certain classical notions and results of the Apollonian theory of conics, such as the parameter or the focus.

Following the rediscovery of ancient mathematics in the I 5 th and 16 th centuries in Europe, arose the problem of understanding, simplifying, restoring and extending one of the most difficult treatises of Greek antiquity, The Conics by Apollonius of Perga. This motivated a great deal of work at the beginning of the i th century, and it can be said that Descartes and Desargues both made very important contributions by introducing two revolutions in the way the practice of geometry was viewed : by reducing it to calculus in Descartes, by founding projective geometry in Desargues ${ }^{6}$. It goes without saying that, in the eyes of their contemporaries, the testing of these innovations must involve a confrontation with the central propositions of Apollonius' treatise. It is to this end that the last pages ${ }^{7}$ of the Draft, which we will analyse here, are devoted. Desargues' writing is often confused and obscure in style. It turns out, however, that once one accepts that Desargues reasons projectively, certain passages that until then had remained misunderstood or curiously ignored, even by the best specialists in the subject, make sense and show us a mathematician who practised geometry as no one else would do after him for almost two centuries.

We will begin this article with a brief review of the contents of the first twenty pages of the text, emphasising the notions of tree, involution and traversal and recalling some of the important results that Desargues proves there. We will then describe a short passage in which the Lyonnais seems to initiate a theory of pairs of circles or conics, which seems unrelated to the Apollonian theory he tackles just afterwards but which, as we will see later, has its place here. We will then analyse the brief and elegant theory of the parameter developed in the Draft, which proceeds by successive specialisations, showing how this notion fits into the projective theory of conics. The foci are then studied by Desargues in an original way, by connecting them to his theory of the parameter and introducing them by a reciprocal of proposition 45 of book III of the Conics of Apollonius, a proposition that the Lyonnais qualifies as obvious and from which all the classical results concerning them easily follow.

It is in the following section, where we analyse how Desargues deals with the hyperbola, that Desargues' projective ways will be strikingly apparent. We will see, among other things, that he does not hesitate to consider the biratio of points situated on the

5. Advice for line 3 on page 16.

6. We refer to the chapter Style cartésien, style arguésien of Gilles-Gaston Granger's book [ı] for a less caricatured analysis.

7. More precisely pages 23 to 29 of the original, see below. 
line at infinity. Before concluding the Draft with general considerations that we will not go into here, the Lyonnais gives an application of his methods to a very beautiful construction of a perspectival nature that echoes the last sentence of his little 1636 pamphlet on perspective ${ }^{8}$ : given a conic cut by a line and a point on that line, he constructs a perspective to another plane so that the figure becomes that of a conic, its major axis and one of its foci.

This article is the last in a series of four (see [ I ], [2] and [3] for the first three) that the authors have devoted to as complete an analysis as possible of the mathematical content of the Draft Project. This work should lead to an annotated republication of the Lyonnais' treatise which will allow the greatest number of people to have access to the extraodinary ideas and methods found in it and to remove certain misunderstandings which still weigh on this text.

\section{Bref résumé des 23 premières pages du Brouillon Pro- ject}

Le Brouillon project d'une atteinte aux événements des rencontres d'un cône et d'un plan de Desargues, paru en I639, est un texte très dense qui compte une trentaine pages auxquelles s'ajoutent quelques pages d'errata et d'ajouts ${ }^{9}$. Le seul exemplaire connu est conservé à la Bibliothèque nationale de France, dans la réserve des livres rares, sous la cote RESM-V-276. C'est à elle que nous ferons référence pour toutes les citations utilisées dans cet article ${ }^{\text {Io }}$. Le lecteur pourra aussi se reporter à l'édition des ouvres mathématiques de Desargues par René Taton dans [14], ainsi qu'aux traductions ou éditions commentées en allemand par Max Zacharias dans [6], en anglais par Judith Field et Jeremy Gray dans [9] et en italien par Fabio Zanin dans [7]. Pour une analyse détaillée de ce qui n'est que résumé dans cette section, nous renvoyons aux articles [I] et [3] ainsi qu'à la prépublication [2].

Le Brouillon commence par l'introduction d'éléments à l'infini permettant d'unifier les notions de droites concourantes et de droites parallèles sous la notion de droites de même ordonnance. Le but d'une ordonnance est alors le point de concours des droites de l'ordonnance. Il est à distance finie si ces droites sont concourantes, et à distance infinie si elles sont parallèles. L'auteur consacre ensuite une dizaine de pages à la notion d'involution. Pour définir celle-ci, qui concerne des couples de points disposés d'une manière particulière sur une droite, il commence par introduire la notion d'arbre.

Soient $\tau$ une droite, que Desargues appelle ici un tronc, et $\mathrm{A}$ un point de $\tau$, ou souche. Trois couples de points de $\tau$, ou noends, B, H; C, G; D, F sont dits former un arbre de souche $\mathrm{A}$ si l'on a les égalités de rectangles suivantes :

$$
\mathrm{AB} \cdot \mathrm{AH}=\mathrm{AC} \cdot \mathrm{AG}=\mathrm{AD} \cdot \mathrm{AF} .
$$

8. Entitled The example of one of the ways of the $S^{r}$. G. D. L. touching the practice of perspective, Without employing any third point of distance or other nature which is out of the field of the Work. Available on the site GALLICA.

9. Les Advertissements.

ı. Ce document est disponible sur le site GALLICA. 
Desargues démontre que la donnée d'un tel arbre fait que les trois couples de nœuds forment ce qu'il appelle une involution, au sens où les égalités de rapports suivantes sont satisfaites :

$$
\frac{\mathrm{GD} \cdot \mathrm{GF}}{\mathrm{CD} \cdot \mathrm{CF}}=\frac{\mathrm{GB} \cdot \mathrm{GH}}{\mathrm{CB} \cdot \mathrm{CH}}, \frac{\mathrm{FC} \cdot \mathrm{FG}}{\mathrm{DC} \cdot \mathrm{DG}}=\frac{\mathrm{FB} \cdot \mathrm{FH}}{\mathrm{DB} \cdot \mathrm{DH}}, \frac{\mathrm{HC} \cdot \mathrm{HG}}{\mathrm{BC} \cdot \mathrm{BG}}=\frac{\mathrm{HD} \cdot \mathrm{HF}}{\mathrm{BD} \cdot \mathrm{BF}} .
$$

Réciproquement, si trois couples de nœuds forment une involution, alors il existe une souche dont ils forment l'arbre.

La donnée d'une involution de Desargues est équivalente à la donnée d'une homographie involutive du tronc $\tau$ vu comme droite projective. Si cette homographie est hyperbolique, ses deux points fixes sont ce que Desargues appelle des nœuds moyens doubles. Ainsi écrit-il dans ce cas que B, H; C, C; D, D est une involution de quatre points $\mathrm{B}, \mathrm{H}, \mathrm{C}, \mathrm{D}$, auquel cas B, H; C, D forment une division harmonique. La souche de l'arbre correspondant est le milieu du segment $[\mathrm{CD}]$. Les nouds accouplés $\mathrm{B}, \mathrm{H}$, n'étant pas moyens, sont dits extrêmes. Si l'homographie est elliptique, elle n'admet pas de point fixe mais il existe cependant un couple de nouds D, F tel que le milieu de [DF] est encore souche de l'arbre correspondant. Les deux nouds D, F sont dits moyens simples. Notons enfin qu'une fois que l'on s'est donné trois couples de points en involution sur une droite, on peut en rajouter autant qu'on le souhaite : la notion est agrégative au même sens que l'est la relation d'alignement pour trois points ${ }^{11}$.

Desargues démontre ensuite que la configuration d'involution est invariante par perspective, ce qui constitue le théorème de la ramée. Si $\tau$ et $\tau^{\prime}$ sont deux troncs et si $\mathrm{K}$ est un point pris hors de ces deux troncs, alors l'image de toute involution sur $\tau$ par projection centrale depuis $\mathrm{K}$ vers $\tau^{\prime}$ est une involution de $\tau^{\prime}$. De fait, l'involution peut se décrire au travers de certaines identités de birapports, et le birapport est un invariant projectif.

Il introduit ensuite sa terminologie sur les coniques et définit la notion de traversale d'un point eu égard à une conique. Celle-ci est équivalente à la notion de polaire. $\mathrm{Si} \mathcal{C}$ est une conique et si $\mathrm{F}$ est un point du plan de la conique, alors il existe une droite $\tau$, dite traversale de $\mathrm{F}$ en égard à la conique $\mathrm{C}$, qui est telle que si $\omega$ est une droite quelconque passant par $\mathrm{F}$ coupant la conique en les points $\mathrm{X}, \mathrm{Y}$ et la traversale $\tau$ en le point $\mathrm{D}$, alors $\mathrm{F}, \mathrm{D} ; \mathrm{X}, \mathrm{X} ; \mathrm{Y}, \mathrm{Y}$ est une involution. Une droite telle $\omega$ passant par $\mathrm{F}$ est appelée une ordonnée de la traversale $\tau$, et le point $\mathrm{D}$ est le point traversal de l'ordonnée $\omega$.

Ces notions généralisent la notion apollonienne de diamètre et d'ordonnée au cas où ces dernières ne sont plus seulement parallèles mais peuvent être concourantes. En effet, si le point $\mathrm{F}$ ci-dessus s'en va à l'infini, toutes les droites passant par $\mathrm{F}$ deviennent parallèles, les points $\infty, \mathrm{D} ; \mathrm{X}, \mathrm{Y}$ forment alors une division harmonique et $\mathrm{D}$ devient le milieu du segment $[\mathrm{XY}]$.

Desargues démontre ensuite toutes les propriétés connues de la polarité projective des coniques :

- toute droite est traversale d'un point;

- les traversales de trois points alignés sont concourantes, et réciproquement;

- une droite tangente à la conique est traversale du point de contact avec celle-ci;

- un diamètre est traversale d'un point à distance infinie;

I I. Voir [3]. 
- le centre de la conique a pour traversale la droite à l'infini du plan;

- la conique induit sur toute droite du plan autre que ses tangentes une involution qui est hyperbolique si la droite rencontre la conique en deux points, et elliptique sinon;

- si d'un point on peut mener deux tangentes à la conique, sa traversale est la droite reliant leurs points de contact avec celle-ci;

- dans le cas général, la traversale d'un point se construit à partir d'un quadrilatère inscrit sur la conique.

La théorie de la traversale est fondée sur le résultat central du Brouillon Project, le théorème d'involution de Desargues. Si B, C, D, E sont quatre points coplanaires en position générale, ils forment les bornes du quadrilatère ainsi défini. Les six diagonales de ce dernier, ou bornales, sont naturellement accouplées de la manière suivante : $(\mathrm{BC}),(\mathrm{DE}) ;(\mathrm{BD}),(\mathrm{CE}) ;(\mathrm{BE}),(\mathrm{DC})$ et le théorème d'involution peut s'énoncer ainsi :

Théorème I.I. - Soient $\mathrm{C}$ une conique et $\mathrm{B}, \mathrm{C}, \mathrm{D}, \mathrm{E}$ quatre points situés sur cette conique. Soit en outre $\tau$ une droite générique du même plan que C. Elle coupe les six bornales du quadrilatère $\mathrm{B}, \mathrm{C}, \mathrm{D}, \mathrm{E}$ en six points accouplés $\mathrm{I}, \mathrm{K} ; \mathrm{P}, \mathrm{Q} ; \mathrm{G}, \mathrm{H}$ et la conique $\mathrm{C}$ en les points L, M. Les points I, K; P, Q; G, H; L, M sont alors en involution.

\section{Souches communes à plusieurs arbres et configura- tions de deux cercles}

Nous commençons notre analyse du Brouillon Project à la ligne 37 de sa page 23. Desargues y considère des involutions induites par deux cercles ou, plutôt, par tout un faiscean linéaire de cercles (du fait de l'agrégativité de la notion d'involution).

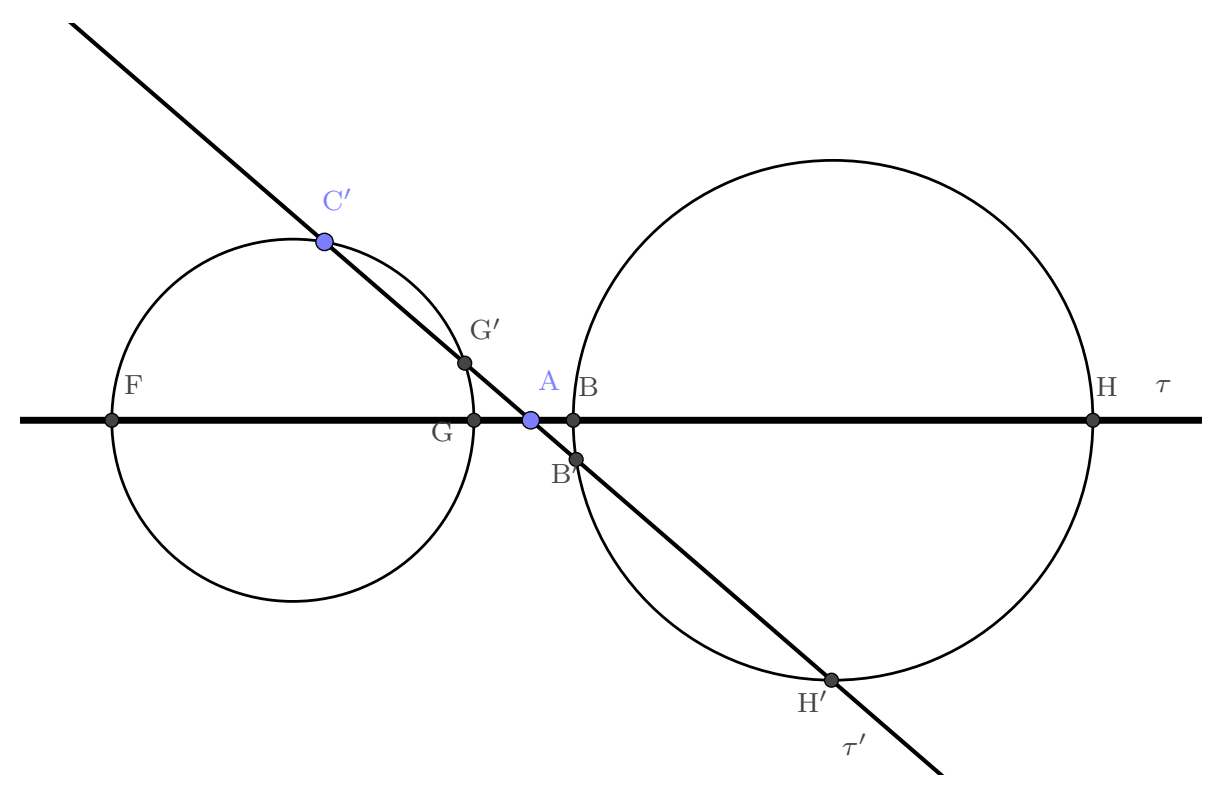

FIGURE I - Deux cercles de diamètres CG, BH et les involutions induites. 
Supposons pour commencer que l'on se donne une droite ou tronc $\tau$ et supposons donnés deux couples de points B, H; C, G d'un arbre de souche A (voir la figure I) de sorte que $\mathrm{AB} . \mathrm{AH}=\mathrm{AC}$.AG. Traçons alors les deux cercles $\mathcal{C}_{\mathrm{BH}}$ et $\mathcal{C}_{\mathrm{CG}}$ de diamètres respectifs les segments $[\mathrm{BH}]$ et $[\mathrm{CG}]$. La condition arboricole $\mathrm{AB} \cdot \mathrm{AH}=\mathrm{AC}$.AG peut se lire comme disant que les puissances de A par rapport à ces deux cercles sont égales. Soit maintenant $\tau^{\prime}$ une droite quelconque passant par $\mathrm{A}$ et coupant le cercle $\mathcal{C}_{\mathrm{BH}}$ (resp. $\mathcal{C}_{\mathrm{CG}}$ ) en les points $\mathrm{B}^{\prime}, \mathrm{H}^{\prime}$ (resp. $\mathrm{C}^{\prime}, \mathrm{G}^{\prime}$ ). La proposition 35 du livre III des Éléments entraine alors que $\mathrm{AB}^{\prime} . \mathrm{AH}^{\prime}=\mathrm{AC}^{\prime} \mathrm{AG}^{\prime}$ et le point $\mathrm{A}$ est donc souche commune à plusieurs arbres ${ }^{\mathrm{I}}$.

Desargues écrit alors : «Outre qu'au lieu de cercles, il peut y avoir sur les mêmes pièces d'entre les deux mêmes de ces quatres nœuds $\mathrm{C}, \mathrm{G} ; \mathrm{B}, \mathrm{H}$, deux quelconques autres coupes de rouleau ${ }^{13}$ disposées en certaine façon que leurs bords opèrent la même chose que ceux des cercles évidemment, au moyen d'une ramée de cet arbre $\mathrm{BH}$. »Cet énoncé assez mystérieux laisse penser qu'il s'agit de l'énoncé analogue pour deux coniques «semblables et semblablement engagées » pour reprendre la terminologie de Michel Chasles ${ }^{14}$. L'idée d'une construction de nature perspectiviste induite par la mention d'une ramée et menant à passer du cas de deux cercles à celui de deux coniques n'est pas sans rappeler ce qu'Oldenburg mentionne, dans sa lettre à Leibniz du 6 avril ı673, du contenu du traité perdu de Desargues intitulé Leçons de Ténèbres. On pourra consulter à ce sujet la note [8] de Gustaf Eneström, ou encore directement ladite lettre, p. 87 \& p. 88 de [I I]. Il semblerait que Desargues y développe une manière de traiter ce sujet au moyen d'une projection centrale d'une sphère vers l'un de ses plans tangents, depuis l'un de ses points intérieurs.

Aux lignes 48 à 58 de la page 23 , il reprend cette construction dans le cas d'une involution de 4 points seulement, involution ayant ici des nœuds moyens doubles. Soit toujours donné le tronc $\tau$ et supposons que H, G, B, F soient quatre points en involution. Ils forment en particulier une division harmonique et l'on dispose alors de deux involutions, en choisissant ceux des nœuds qui seront moyens doubles. Nous pouvons considérer l'involution B, H; G, G; F, F, dont A (le milieu du segment [GF]) est souche de l'arbre correspondant, et l'involution B, B; H, H; F, G, dont L (le milieu du segment $[\mathrm{BH}])$ est souche, dite réciproque ${ }^{\mathrm{Is}}$ de la précédente. Les cercles $\mathcal{C}_{\mathrm{FG}}$ et $\mathcal{C}_{\mathrm{BH}}$ coupent toute droite issue aussi bien de $\mathrm{A}$ que de $\mathrm{L}$ selon quatre points en involution dont l'arbre associé a pour souche le point A ou le point L. Comme pour le cas précédemment étudié, Desargues annonce que l'on peut généraliser ce résultat à un couple de coniques bien choisies.

La fin de ce passage est consacrée au cas analogue construit sur la base d'une involution elliptique, c'est-à-dire à nœuds moyens simples plutôt que doubles ${ }^{16}$. Soit donc $\tau$ un tronc et $\mathrm{H}, \mathrm{B} ; \mathrm{C}, \mathrm{G} ; \mathrm{D}, \mathrm{F}$ des points en involution de sorte que $\mathrm{C}, \mathrm{G}$ et $\mathrm{D}, \mathrm{F}$ soient des couples de nœuds moyens simples, et $\mathrm{B}, \mathrm{H}$ un couple de nœuds extrêmes. Les points $D$ et $G$ sont confondus, de même que $F$ et $C$, et le point $A$, milieu de [FG], est souche de l'arbre correspondant. Traçons maintenant le cercle $\mathcal{C}_{\mathrm{FG}}$ de diamètre $[\mathrm{FG}]$, donc de

I 2. p. $23,1 \cdot 37-42$

I3. ou conique

I4. Michel Chasles, Géométrie de situation. Mémoire sur les propriétés de systèmes de sections coniques, situées dans un même plan, Annales de mathématiques pures et appliqués, tome I 8 (I 827-1 828), p. 277-30 I.

I 5. p.9, 1.4

16. Cela découle de l'engagement de la souche A d'entre les nouds F et F, voir p. 23, 1. 6o. 
centre $A$, et le cercle $\mathcal{C}_{\mathrm{AB}}$ de diamètre $[\mathrm{AB}]$, voir la figure 2.

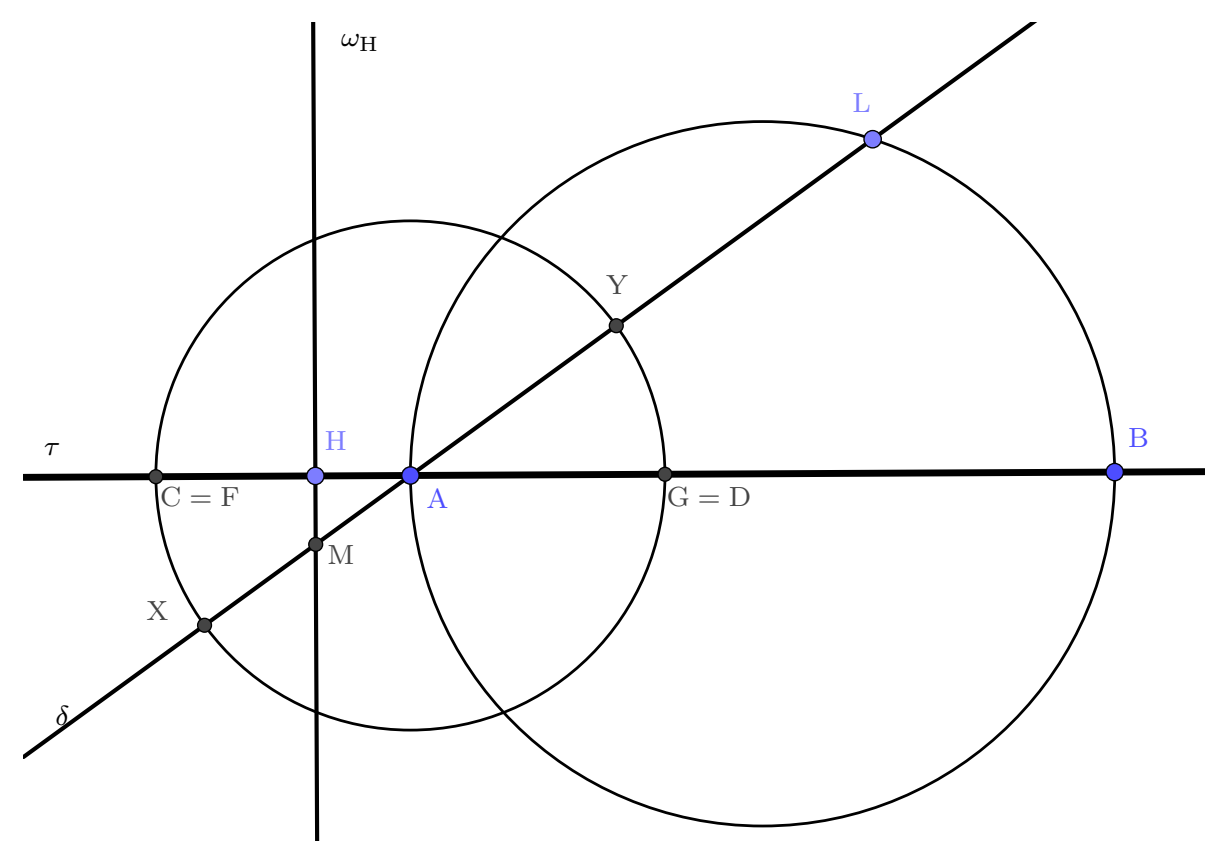

FIGURE 2 - Le deuxième cas : involution elliptique.

Soit $\omega_{\mathrm{H}}$ la droite perpendiculaire à $\tau$ passant par $\mathrm{H}$ et soit $\delta$ une droite quelconque passant par $A$. Elle coupe $\mathcal{C}_{\mathrm{AB}}$ en les points $L$ et $A, \mathcal{C}_{\mathrm{FG}}$ en les points $\mathrm{X}$ et $\mathrm{Y}$, et enfin $\omega_{\mathrm{H}}$ en M. Alors X, Y; L, M sont des couples de nouds d'un arbre de souche A. En effet, étant donné que $\mathrm{AG} \cdot \mathrm{AF}=\mathrm{AH}$.AB et que par ailleurs $\mathrm{F}, \mathrm{G}$ et $\mathrm{X}, \mathrm{Y}$ sont des couples de points diamétralement opposés sur le même cercle de centre $A$, on a aussi $\mathrm{AG} \cdot \mathrm{AF}=\mathrm{AX}$.AY. Mais les deux triangles rectangles AHM et ALB sont semblables et donc

$$
\frac{\mathrm{AH}}{\mathrm{AM}}=\frac{\mathrm{AL}}{\mathrm{AB}},
$$

ainsi $\mathrm{AH} . \mathrm{AB}=\mathrm{AL} . \mathrm{AM}$, ce qui démontre l'assertion de Desargues. Remarquons pour finir que si l'on trace le cercle $\mathcal{C}_{\mathrm{BH}}$ de diamètre $[\mathrm{BH}]$, alors celui-ci coupe le cercle $\mathrm{C}_{\mathrm{CG}}$ et que A est le pied de l'axe radical de ces deux cercles. On peut se poser la question de savoir pourquoi Desargues considère la droite $\omega_{\mathrm{H}}$ perpendiculaire à $\tau$ en $\mathrm{H}$ plutôt que l'axe radical. La réponse se trouve bien avant dans le Brouillon, aux lignes 9 et suivantes de la page 9, où Desargues dit que $H$ est pour souche aux deux couples de nœuds G, F et $\mathrm{B}, \mathrm{A}$. La construction est alors strictement similaire à celle faite dans le cas précédent basé sur une involution hyperbolique : la droite $\omega_{\mathrm{H}}$ est l'axe radical des deux cerles $\mathcal{C}_{\mathrm{AB}}$ et $\mathcal{C}_{\mathrm{FG}}$.

Comme pour le cas précédent, Desargues mentionne une généralisation au cas de deux coniques, «dont une ramée fait voir la vérité ». 


\section{La théorie du paramètre}

Dans la dernière partie du Brouillon Project, Desargues met en œuvre les outils qu'il a développés jusque là (l'involution, la traversale, les méthodes projectives) pour donner un rapide et élégant traitement de la théorie apollonienne du paramètre et des foyers. Cela lui permet en quelque sorte de justifier l'intérêt des méthodes nouvelles qu'il propose auprès de ses contemporains.

Â la page 24, des lignes i 6 à 39, il énonce un long théorème qu'il démontrera dans les pages suivantes. Il procède par spécialisations successives, en partant d'une conique coupée par une droite quelconque pour arriver à une conique coupée par un de ses diamètres, ce qui lui donnera une caractérisation du paramètre, et enfin à une conique coupée par son grand axe, ce qui lui permettra de retrouver rapidement les énoncés concernant les foyers. Nous allons diviser ce long énoncé en plusieurs propositions afin d'en faciliter la compréhension.

Donnons-nous une conique $\mathcal{C}$ et une droite $\tau^{17}$ coupant la conique $\mathcal{C}$ en les points L et M. Soient B, C, D, E quatre points de la conique tels que $(\mathrm{BC})$ soit parallèle à (DE). Nommons I l'intersection de (BC) avec $\tau$ et $\mathrm{K}$ celle de (DE) avec $\tau$. Soit $\delta_{\mathrm{L}}$ une droite quelconque passant par $\mathrm{L}$ et notons $\mathrm{R}, \mathrm{S}$ les points d'intersection de $\delta_{\mathrm{L}}$ avec $(\mathrm{BC})$ et (DE) respectivement, voir la figure 3. La première proposition donnée par Desargues s'énonce ainsi :

Proposition 3.I. - Sous les hypothèses ci-dessus, on a l'égalité de rapports suivante:

$$
\frac{\mathrm{KS} \cdot \mathrm{KM}}{\mathrm{KD} \cdot \mathrm{KE}}=\frac{\mathrm{IR} \cdot \mathrm{IM}}{\mathrm{IC} \cdot \mathrm{IB}}
$$

et Desargues qualifie le rectangle IR.IM (resp. IC.IB) de relatif $d u$ rectangle KS.KM (resp. KD.KE).

La très jolie symétrie de cette identité nous amène à l'interpréter ainsi : fixons une droite (BC) coupant la conique en $\mathrm{B}$ et $\mathrm{C}$ et la transversale $\tau$ en I. Alors, quelle que soit la droite $(\mathrm{DE})$ parallèle à $(\mathrm{BC})$, coupant $\mathcal{C}$ en $\mathrm{D}, \mathrm{E}$ et $\tau$ en $\mathrm{K}$, on a l'égalité de rapports cidessus. Cela n'est pas sans rappeler les énoncés sur les cordes parallèles qu'on trouve par exemple aux propositions i6 et suivantes du livre III des Coniques d'Apollonius. Néanmoins, la proposition de Desargues en est bien différente de par l'utilisation de la droite arbitraire $\delta_{\mathrm{L}}$. Nous verrons cependant qu'au cours de sa démonstration, Desargues redémontre sans le dire la proposition d'Apollonius. Claude Mydorge, dans le livre IV de son Prodromi Catoptricorum et Dioptricorum, paru en I637, a d'ailleurs donné de cette proposition une démonstration simple et une application intéressante au problème de la détermination d'une conique passant par 5 points ${ }^{18}$.

Supposons maintenant que la droite $\delta_{\mathrm{L}}$ soit tracée de sorte que l'on ait KS.KM = $\mathrm{KE} . \mathrm{KD}$, égalité que l'on peut réécrire sous la forme $\mathrm{KS}=(\mathrm{KE} . \mathrm{KD}) / \mathrm{KM}$, dont le second membre est donné et ne dépend pas de $\delta_{\mathrm{L}}$. Soit par ailleurs $\omega_{\mathrm{M}}$ la droite parallèle à (BC) passant par M et notons $\mathrm{T}$ son intersection avec $\delta_{\mathrm{L}}$. La deuxième proposition donnée par Desargues s'énonce ainsi :

I7. Dans l'orginal : «Quand en un plan une droite $\mathrm{PH}$, comme tronc etc. 》

I 8. voir [I 2], p. 385 et suivantes. 


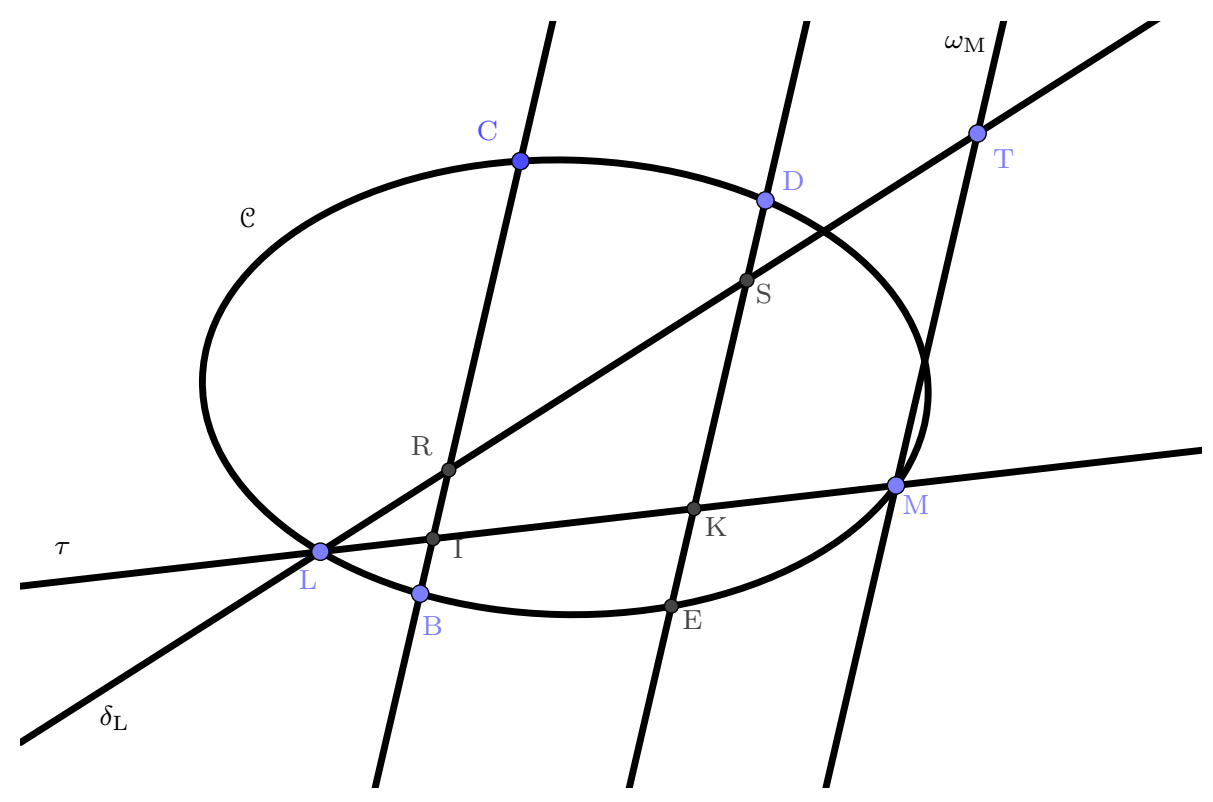

FIGURE 3 - La situation des deux premières propositions sur le paramètre

Proposition 3.2. - Sous les hypothèses ci-dessus, on a l'égalité de rapports suivante:

$$
\frac{\mathrm{KL} \cdot \mathrm{KM}}{\mathrm{KE} \cdot \mathrm{KD}}=\frac{\mathrm{ML}}{\mathrm{MT}}
$$

La dernière proposition énoncée dans ce long passage introduit alors le paramètre :

Proposition 3.3. - Supposons qu'outre les hypothèses faites jusqu'ici la droite $\tau$ soit un diamètre de $\mathrm{C}$ et que $(\mathrm{BC}),(\mathrm{DE})$ et $\omega_{\mathrm{M}}$ en soient des ordonnées. Alors $\mathrm{MT}$ est le paramètre de la conique $\mathrm{C}$ en égard an diamètre $\tau$.

Desargues précisera le contenu de cette proposition et justifiera l'appellation de paramètre qu'il donne au segment MT après la démonstration des deux premières propositions, qui occupe les lignes 40 à 60 de la page 24 .

Reprenons les hypothèses de la première proposition et rappelons qu'il s'agit de démontrer que

$$
\frac{\mathrm{KS} \cdot \mathrm{KM}}{\mathrm{KD} \cdot \mathrm{KE}}=\frac{\mathrm{IR} \cdot \mathrm{IM}}{\mathrm{IC} \cdot \mathrm{IB}}
$$

Du fait du parallèlisme de (BC) et (DE), on a en effet

$$
\frac{\mathrm{IL}}{\mathrm{IR}}=\frac{\mathrm{KL}}{\mathrm{KS}}
$$

et donc, bien évidemment :

$$
\frac{\mathrm{IL} \cdot \mathrm{IM}}{\mathrm{IR} \cdot \mathrm{IM}}=\frac{\mathrm{KL} \cdot \mathrm{KM}}{\mathrm{KS} \cdot \mathrm{KM}}
$$

soit, en alternant :

$$
\frac{\mathrm{KL} \cdot \mathrm{KM}}{\mathrm{IL} \cdot \mathrm{IM}}=\frac{\mathrm{KS} \cdot \mathrm{KN}}{\mathrm{IR} \cdot \mathrm{IM}} .
$$


Desargues écrit alors qu'on a par ailleurs démontré que

$$
\frac{\mathrm{KL} . \mathrm{KM}}{\mathrm{IL} \cdot \mathrm{IM}}=\frac{\mathrm{KD} \cdot \mathrm{KE}}{\mathrm{IC} \cdot \mathrm{IB}},
$$

ce qui mérite quelque explication, que nous donnons ici : notons $\mathrm{P}$ et $\mathrm{Q}$ les intersections respectives des deux autres bornales $(\mathrm{BE})$ et $(\mathrm{DC})$ du quadrangle $\mathrm{BCDE}$ avec la transversale $\tau$. D'après le théorème d'involution de Desargues ${ }^{19}$, les points $\mathrm{P}, \mathrm{Q} ; \mathrm{L}, \mathrm{M} ; \mathrm{I}, \mathrm{K}$ forment une involution et donc

$$
\frac{\mathrm{KL} \cdot \mathrm{KM}}{\mathrm{IL} \cdot \mathrm{IM}}=\frac{\mathrm{KQ} \cdot \mathrm{KP}}{\mathrm{IQ} \cdot \mathrm{IP}} .
$$

Or Desargues a démontré ${ }^{20}$ que, dans le cas où deux bornales sont parallèles, on a aussi

$$
\frac{\mathrm{KQ} \cdot \mathrm{KP}}{\mathrm{IQ} \cdot \mathrm{IP}}=\frac{\mathrm{KD} \cdot \mathrm{KE}}{\mathrm{IC} \cdot \mathrm{IB}},
$$

d'où suit bien que

$$
\frac{\mathrm{KL} . \mathrm{KM}}{\mathrm{IL} \cdot \mathrm{IM}}=\frac{\mathrm{KD} \cdot \mathrm{KE}}{\mathrm{IC} \cdot \mathrm{IB}},
$$

ce qui constitue l'énoncé classique d'Apollonius sur les cordes parallèles cité plus haut. De l'identité I on tire alors

$$
\frac{\mathrm{KL} \cdot \mathrm{KM}}{\mathrm{IL} \cdot \mathrm{IM}}=\frac{\mathrm{KD} \cdot \mathrm{KE}}{\mathrm{IC} \cdot \mathrm{IB}}=\frac{\mathrm{KS} \cdot \mathrm{KM}}{\mathrm{IR} \cdot \mathrm{IM}}
$$

soit, en alternant :

$$
\frac{\mathrm{KS} \cdot \mathrm{KM}}{\mathrm{KE} \cdot \mathrm{KD}}=\frac{\mathrm{IR} \cdot \mathrm{IM}}{\mathrm{IC} \cdot \mathrm{IB}},
$$

ce qu'il fallait démontrer.

Si maintenant on suppose en plus que KS.KM $=$ KE.KD, alors il vient immédiatement que

$$
\mathrm{IR} . \mathrm{IM}=\mathrm{IC} . \mathrm{IB}
$$

et en outre que

$$
\frac{\mathrm{KL} \cdot \mathrm{KM}}{\mathrm{KE} \cdot \mathrm{KD}}=\frac{\mathrm{KL} \cdot \mathrm{KM}}{\mathrm{KS} \cdot \mathrm{KM}}=\frac{\mathrm{KL}}{\mathrm{KS}} .
$$

Or, du fait du parallèlisme de DE et BC,

$$
\frac{\mathrm{KL}}{\mathrm{KS}}=\frac{\mathrm{ML}}{\mathrm{MT}}
$$

et donc

$$
\frac{\mathrm{KL} \cdot \mathrm{KM}}{\mathrm{KE} \cdot \mathrm{KD}}=\frac{\mathrm{ML}}{\mathrm{MT}},
$$

ce qui démontre la deuxième proposition.

I9. p. I6, 1. 56-6I

20. p. $17,1.32-36$ 
Desargues revient alors, p. 24 1. 6I, à la troisième proposition, dont il dit qu'elle est évidente sans préciser outre mesure. On peut essayer de comprendre pourquoi il insiste sur cette evidence. Supposons que l'on place l'ordonnée ED de sorte qu'elle passe par le centre $\mathrm{K}$ de la conique. D'après la deuxième proposition on a

$$
\frac{\mathrm{ML}}{\mathrm{MT}}=\frac{\mathrm{KL} \cdot \mathrm{KM}}{\mathrm{KE} \cdot \mathrm{KD}}
$$

ce qui, du fait que $\tau$ est un diamètre et que $\mathrm{BC}, \mathrm{DE}$ etc. sont ses ordonnées, donne

$$
\frac{\mathrm{ML}}{\mathrm{MT}}=\frac{\mathrm{KM}^{2}}{\mathrm{KD}^{2}}
$$

soit encore

$$
\mathrm{MT}=\mathrm{ML} \cdot \frac{\mathrm{KD}^{2}}{\mathrm{KM}^{2}}=2 \mathrm{KM} \frac{\mathrm{KD}^{2}}{\mathrm{KM}^{2}}=2 \frac{\mathrm{KD}^{2}}{\mathrm{KM}} .
$$

Ainsi, lorsque $\tau$ est le grand axe de la conique, MT est égal à deux fois le carré du demi grand axe divisé par le demi petit axe, ce qui est une définition classique du paramètre.

Il va cependant préciser tout cela après un a parte sur les droites parallèles et les points à l'infini, où il se libère complètement des précautions faites au début du Brouillon Project concernant les buts d'ordonnances qui deviennent ici de véritables points à distance infinie ${ }^{21}$ :

«De ce qui est dit ci-devant on aura conçu que pour mener d'un quelconque point une droite d'une même ordonnance avec deux parallèles entre elles, cela s'entend que cette droite soit menée aussi parallèle à ces deux, \& de même que pour mener d'un quelconque point une droite à un point à distance infinie en une autre droite, cela s'entend qu'il faut mener cette droite parallèle à celle où le point assigné est à distance infinie. 》

Conscient sans doute qu'il n'a pas donné tous les éléments permettant de convaincre ses lecteurs qu'il a bien défini le paramètre d'une conique, Desargues va en donner une théorie concise mais complète des lignes 8 à 56 de la page 25 , en commençant par la phrase suivante :

«Encore que ce qui suit paroisse évidemment des choses cy devant demonstrées, neantmoins : 》

Il change le lettrage des points : soient $\mathcal{C}$ une conique, $\delta$ un de ses diamètres, coupant $\mathcal{C}$ en $\mathrm{E}$ et $\mathrm{C}$, et passant par son centre 7 . Traçons les ordonnées respectives $\omega_{\mathrm{E}}, \omega_{7}$ et $\omega_{\mathrm{C}}$, de sorte que $\omega_{\mathrm{E}}$ et $\omega_{\mathrm{C}}$ sont des tangentes à la conique, et que $\omega_{7}$ est le diamètre conjugué de $\delta$. Traçons sur $\omega_{\mathrm{E}}$ le paramètre $\mathrm{EF}$ de $\mathcal{C}$ eu égard au diamètre $\delta$.

Desargues donne, dans les Advertissements, un moyen simple de construire ce paramètre à la règle et au compas, qu'il demande de rajouter après la ligne 26 . En voici le détail : traçons, par E, la bissectrice $\beta$ de l'angle $\widehat{\mathrm{CEB}}$. Cette bissectrice coupe $\mathcal{C}$ en $\mathrm{E}$ et en un autre point $\mathrm{V}$, qui est sur une ordonnée $\omega_{\mathrm{W}}$ de $\delta$. La droite $(\mathrm{CV})$ coupe alors $\omega_{\mathrm{E}}$ en le point $\mathrm{F}$ recherché. En effet, comme $(\mathrm{WV})$ est parallèle à $(\mathrm{EF})$, les angles $\widehat{\mathrm{EVW}}$ et $\widehat{\mathrm{WEV}}$ sont égaux et donc WV $=\mathrm{WE}$. Il en découle que

$$
\frac{\mathrm{WE} \cdot \mathrm{WC}}{\mathrm{WV} \cdot \mathrm{WV}}=\frac{\mathrm{WC}}{\mathrm{WV}}
$$

2I. p. $25,1.3-8$ 
et le second membre, d'après le théorème de Thalès, est égal à EF/EC. Ainsi a-t-on

$$
\mathrm{EF}=\mathrm{EC} \cdot \frac{\mathrm{WV}}{\mathrm{WC}}
$$

et EF est bien le paramètre d'après la définition qu'en donne Apollonius.

Revenons au corps principal du texte et prenons un point $L$ sur $\mathcal{C}$ par lequel nous faisons passer la tangente $\tau_{\mathrm{L}}$. Celle-ci coupe les ordinales $\omega_{\mathrm{E}}$ et $\omega_{\mathrm{C}}$ en les points $\mathrm{B}$ et $\mathrm{D}$. La proposition suivante est énoncée après sa démonstration ${ }^{22}$, et donne la définition recherchée par Desargues du paramètre :

Proposition 3.4. - Sous les hypothèses ci-dessus, on a l'égalité suivante:

$$
\mathrm{EB} . \mathrm{CD}=\frac{1}{4} \mathrm{EC} \cdot \mathrm{EF},
$$

et le second membre est indépendant du point $\mathrm{L}$ choisi sur la conique.

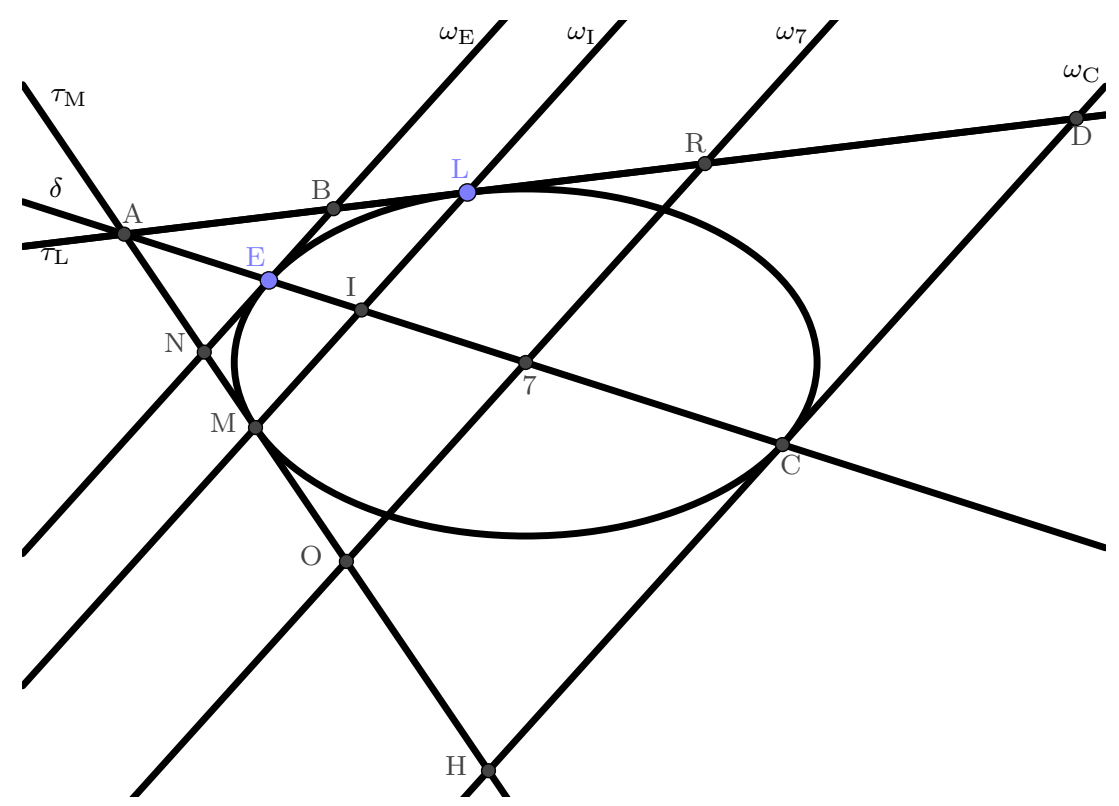

FIGURE 4 - La situation de la quatrième proposition.

Complétons les données de la proposition ci-dessus par des points et droites qui nous seront utiles. Traçons par L l'ordonnée $\omega_{\mathrm{I}}$ eu égard au diamètre $\delta$. Celle-ci coupe $\mathcal{C}$ en $\mathrm{L}$ mais aussi en un autre point M. Les tangentes $\tau_{\mathrm{L}}, \tau_{\mathrm{M}}$ et le diamètre $\delta$ sont concourants en un point A. Notons enfin $\mathrm{N}$ (resp. O et H) l'intersection de $\tau_{\mathrm{M}}$ et de $\omega_{\mathrm{E}}$ (resp. de $\tau_{\mathrm{M}}$ et de $\omega_{7}$, de $\tau_{\mathrm{M}}$ et de $\omega_{\mathrm{C}}$ ).

Les quatre ordonnées $\omega_{\mathrm{E}}, \omega_{\mathrm{I}}, \omega_{7}$ et $\omega_{\mathrm{C}}$ sont de même ordonnance, avec un but à l'infini. Par construction, $(\mathrm{LM})=\omega_{\mathrm{I}}$ est traversale du point $\mathrm{A}$ eu égard à $\mathrm{C}$ et donc E, E; C, C; I, A sont des couples de nouds d'une involution. Comme 7 est le milieu du segment des nœuds moyens doubles $\mathrm{E}$ et $\mathrm{C}$, il est souche de l'arbre correspondant. Cela

22. p. $25,1.54$ 
entraîne que E, C; I, A est une division harmonique et donc, par les propriétés générales des arbres, que A est souche d'un arbre dont E, C; 7, I sont des couples de nœuds. On a donc $\mathrm{A} 7 . \mathrm{AI}=\mathrm{AE} . \mathrm{AC}$, soit encore

$$
\frac{\mathrm{A} 7}{\mathrm{AC}}=\frac{\mathrm{AE}}{\mathrm{AI}}
$$

Par une ramée au but des ordonnées, A est aussi souche d'un arbre dont B, D; R, L sont des couples de nœuds, et d'un autre arbre encore dont N, H; M, O sont des couples de nœuds ${ }^{23}$. D'après le théorème de Thalès, on a

$$
\frac{\mathrm{A} 7}{\mathrm{AE}}=\frac{7 \mathrm{R}}{\mathrm{EB}} \text { et } \frac{\mathrm{AC}}{\mathrm{AI}}=\frac{\mathrm{CD}}{\mathrm{IL}},
$$

d'où l'on tire

$$
\frac{7 \mathrm{R}}{\mathrm{EB}}=\frac{\mathrm{CD}}{\mathrm{IL}}
$$

soit finalement

$$
\text { 7R.IL }=\text { EB.CD. }
$$

Desargues énumère ensuite ${ }^{24}$ toute une série d'identités de rapports qui sont conséquences du théorème de Thalès :

$$
\frac{\mathrm{CD}}{\mathrm{CH}}=\frac{7 \mathrm{R}}{7 \mathrm{O}}=\frac{\mathrm{IL}}{\mathrm{IM}}=\frac{\mathrm{EB}}{\mathrm{EN}}
$$

et

$$
\frac{\mathrm{AL}}{\mathrm{AD}}=\frac{\mathrm{A} 7}{\mathrm{AR}}=\frac{\mathrm{AI}}{\mathrm{AL}}=\frac{\mathrm{AE}}{\mathrm{AB}}=\frac{\mathrm{AH}}{\mathrm{AD}} \text { etc. }
$$

On a bien évidemment ${ }^{25}$ :

$$
\frac{\mathrm{A} 7 \cdot \mathrm{AI}}{\mathrm{AI} \cdot \mathrm{AI}}=\frac{\mathrm{A} 7}{\mathrm{AI}}
$$

et donc, A étant souche de l'arbre E, C; 7, I, on peut appliquer ce qui est énoncé plus haut dans le Brouillon ${ }^{26}$, qui dans ce cas nous donne

$$
\frac{\mathrm{A} 7}{\mathrm{AI}}=\frac{7 \mathrm{E} \cdot 7 \mathrm{C}}{\mathrm{IC} \cdot \mathrm{IE}}
$$

Or d'après le théorème de Thalès on a aussi

$$
\frac{\mathrm{A} 7}{\mathrm{AI}}=\frac{7 \mathrm{R}}{\mathrm{IL}}
$$

et bien-sûr

$$
\frac{7 \mathrm{R}}{\mathrm{IL}}=\frac{7 \mathrm{R} \cdot \mathrm{IL}}{\mathrm{IL} \cdot \mathrm{IL}}=\frac{7 \mathrm{R} \cdot \mathrm{IL}}{\mathrm{IL} \cdot \mathrm{IM}}
$$

\footnotetext{
23. p. $25,1.29$

24. p. $25,1.3 \mathrm{I}-33$

25. p. $25,1.34$

26. p. $4,1.7$
} 
car, $\delta$ étant un diamètre, IL $=\mathrm{IM}$. On peut donc en conclure que

$$
\frac{\mathrm{A} 7 . \mathrm{AI}}{\mathrm{AI} \cdot \mathrm{AI}}=\frac{7 \mathrm{R} \cdot \mathrm{IL}}{\mathrm{IL} \cdot \mathrm{IM}}=\frac{7 \mathrm{E} \cdot 7 \mathrm{C}}{\mathrm{IC} \cdot \mathrm{IE}}
$$

soit, « en changeant ${ }^{27} \gg:$

$$
\frac{7 \mathrm{C} .7 \mathrm{E}}{7 \mathrm{R} . \mathrm{IL}}=\frac{\mathrm{IC} \cdot \mathrm{IE}}{\mathrm{IL} \cdot \mathrm{IM}}
$$

En utilisant l'identité 2, on obtient

$$
\frac{7 \mathrm{E} .7 \mathrm{C}}{\mathrm{EB} \cdot \mathrm{CD}}=\frac{\mathrm{IC} \cdot \mathrm{IE}}{\mathrm{IL} \cdot \mathrm{IM}}
$$

Comme EF est le paramètre de $\mathcal{C}$ eu égard au diamètre $\delta$, on a également

$$
\frac{\mathrm{IC} \cdot \mathrm{IE}}{\mathrm{IL} \cdot \mathrm{IM}}=\frac{\mathrm{EC}}{\mathrm{EF}},
$$

et comme $\delta$ est un diamètre et que 7 est le centre de la conique, on a par ailleurs

$$
\frac{7 \mathrm{E} .7 \mathrm{C}}{\mathrm{EB} \cdot \mathrm{CD}}=\frac{(1 / 4) \mathrm{EC}^{2}}{\mathrm{~EB} \cdot \mathrm{CD}}
$$

d'où suit que

$$
\mathrm{EB} . \mathrm{CD}=\frac{1}{4} \mathrm{EC} \cdot \mathrm{EF},
$$

ce qui démontre la quatrième proposition et achève le développement de la théorie du paramètre dans le Brouillon Project. Il note dans les Advertissements que celui-ci n'est finalement qu'un cas (troisième proposition) d'un cas (deuxième proposition) d'un cas (première proposition), sans préciser de quoi il est ainsi un cas : il s'agit du théorème d'involution bien-sûr, faisant que la théorie apollonienne du paramètre, comme celle des diamètres, s’insère naturellement dans la théorie arguésienne générale de la traversale.

\section{La théorie des foyers}

De la ligne i 7 de la page 25 à la ligne 6 de la page 26, passage que nous citerons intégralement plus loin, Desargues présente brièvement sa théorie des foyers.

Les données sont les mêmes que celles de la proposition 3.4, avec l'hypothèse supplémentaire que $\delta$ est le grand axe de la conique. Traçons le cercle $\mathcal{D}$ de diamètre [BD]. $\mathrm{D}$ 'après le théorème de Thalès, son centre est au point $\mathrm{R}$ d'intersection de l'ordonnée $\omega_{7}$ avec la droite (BD). Il coupe le grand axe $\delta$ en les points $\mathrm{P}$ et $\mathrm{Q}$. Desargues énonce p. 25, 1.6I :

Proposition 4.I. - Sous les hypothèses ci-dessus, on a l'identité suivante:

$$
\text { PE.PC }=\frac{1}{4} \mathrm{EC} \cdot \mathrm{EF} .
$$

\footnotetext{
27. p.25, 1. 40
} 
La démonstration n'est pas donnée par Desargues. Elle est en effet très simple et la voici : comme $[\mathrm{BD}]$ est diamètre du cercle $\mathcal{D}$ et que $\mathrm{Q}$ est sur ce cercle, l'angle $\widehat{\mathrm{BQS}}$ est droit. Comme $\delta$ est grand axe, ses ordonnées $\omega_{\mathrm{E}}=(\mathrm{EB})$ et $\omega_{\mathrm{C}}=(\mathrm{CD})$ y sont perpendiculaires. On a donc $\widehat{\mathrm{CQD}}=\widehat{\mathrm{EBQ}}$ et les deux triangles rectangles $\mathrm{BQE}$ et CQD sont semblables. De même, BPE et PDC sont semblables et ainsi

$$
\frac{\mathrm{PE}}{\mathrm{EB}}=\frac{\mathrm{CD}}{\mathrm{PC}}
$$

soit PE.PC = EB.CD. D'après la proposition 3.4, le second membre est égal à (1/4)EC.EF, ce qui démontre la proposition.

Il écrit ensuite ${ }^{28}$ que ces deux points $\mathrm{P}$ et $\mathrm{Q}$ sont les foyers de la conique car ils satisfont en outre aux propositions suivantes :

Proposition 4.2. - Sous les hypothèses faites ci-dessus, EC $=$ PL + LQ.

Proposition 4.3. - Sous les hypothèses faites ci-dessus, la tangente à la conique en L est bissectrice de l'angle QLP.

Dans les notes de son édition allemande [6] du Brouillon Project ${ }^{29}$, Max Zacharias affirme que ces énoncés découlent d'un dictionnaire savant avec une proposition assez compliquée portant sur le cercle énoncée et démontrée plus haut dans le Brouillon ${ }^{30}$. Notre thèse est toute autre. Citons intégralement ce court passage sur les foyers :

«Et par une converse évidente de ce qui a été démontré, quand la diamétrale comme $\mathrm{E}_{7} \mathrm{C}$, est le grand des essieux de la figure, le brin comme $\mathrm{BD}$, est diamètre d'un cercle dont la circonférence passe en deux points comme Q, \& $\mathrm{P}$, de façon que le rectangle des pièces de cette diamétrale $\mathrm{E}_{7} \mathrm{C}$, contenues entre le quelconque de ces points $\mathrm{P}, \&$ chacun des points comme E, \& $\mathrm{C}$, qu'y donne le bord de la figure, est encore égal au quart du rectangle EC, EF, la pièce comme EC, est égale à la somme ou à la différence des deux droites menées du point d'attouchement comme $\mathrm{L}$, à chacun de ces points comme $\mathrm{P}, \& \mathrm{Q}$, à savoir à la somme ou différence des deux droites menées comme LP, LQ, \& la touchante LD, mypartit un des angles que ces deux droites menées comme QP, PL, font entre elles.

C'est à dire, que ces deux points comme Q, \& $\mathrm{P}$, sont les points nommés Nombrils, Bruslans, ou foyerz, de la figure. »

La question est de savoir pourquoi Desargues commence sa phrase par les mots «Et par une converse évidente » et de savoir ce que l'on déduit de cette converse. «Ce qui a été démonstré » renvoie d'après nous à la proposition 4.I. Sa converse, évidente, est en fait la proposition 45 du livre III des Coniques d'Apollonius, qui nous dit, en reprenant les notations de Desargues, que si PE.PC $=(1 / 4) \mathrm{EC} . \mathrm{EF}$, alors P est intersection du cercle de diamètre $[\mathrm{BD}]$ avec le grand axe. La démonstration de cette proposition est très facile et repose sur des considérations de triangles semblables. Que les points $\mathrm{P}$ et $\mathrm{Q}$ construits ainsi soient les foyers de la conique, c'est-à-dire satisfassent

28. p. 26,1 . I-6

29. René Taton la reprend textuellement dans [ I4].

30. De la ligne 49 de la page i 8 à la ligne 32 de la page 20. 
aux propositions 4.2 et 4.3 , est alors le contenu des propositions 46 à 52 du même livre III, dont les démonstrations sont toutes très simples.

Nous sommes donc convaincus que Desargues entend en fait que ce que nous avons appelé la proposition 4. I est la réciproque de la proposition 45 du livre III des Coniques, qu'il qualifie d'évidente, et dont les conséquences sont que les points qu'il construit sont bien les foyers de la conique.

\section{L'hyperbole et l'emploi des éléments à l'infini}

Des lignes 7 à 37 de la page 26, Desargues va brièvement étudier l'hyperbole. Ses preuves y sont extrêment concises et, à notre connaissance, aucun auteur n'a réellement essayé de les comprendre. Nous verrons que cela est sans doute dû au fait que l'on ne peut les appréhender que si l'on admet que Desargues y raisonne de manière résolument projective, en considérant par exemple des configurations de points sur la droite à l'infini.

Soient $\mathcal{C}$ une hyperbole de centre 7 et $\delta$ l'un de ses diamètres, coupant $\mathcal{C}$ aux points $\mathrm{E}, \mathrm{C}$. Soient $\tau_{\mathrm{E}}$ la tangente en $\mathrm{E}$ et $\tau_{\mathrm{C}}$ celle en $\mathrm{C}$. Notons en outre $\alpha, \alpha^{\prime}$ les deux asymptotes de l'hyperbole; elles rencontrent $\tau_{\mathrm{E}}$ en les points respectifs $\mathrm{X}$ et $\mathrm{Y}$, et $\tau_{\mathrm{C}}$ en les points respectifs $\mathrm{Z}$ et $\mathrm{K}$. Soit $\mathrm{L}$ un point quelconque pris sur l'hyperbole et notons $\tau_{\mathrm{L}}$ la tangente en $\mathrm{L}$, qui rencontre $\tau_{\mathrm{C}}$ en $\mathrm{D}$ et $\tau_{\mathrm{E}}$ en $\mathrm{B}$. La droite $\tau_{\mathrm{L}}$ rencontre en outre le diamètre $\delta$ au point $\mathrm{A}$ et notons $\tau_{\mathrm{M}}$, où $\mathrm{M}$ est sur l'hyperbole, l'autre tangente passant par A. La droite (LM) est donc la traversale de A eu égard à $C$. Notons I le point traversal de l'ordonnée $\delta$ de cette traversale (LM), S le point de rencontre entre (LM) et $\alpha$ et $\mathrm{T}$ le point de rencontre entre (LM) et $\alpha^{\prime}$.

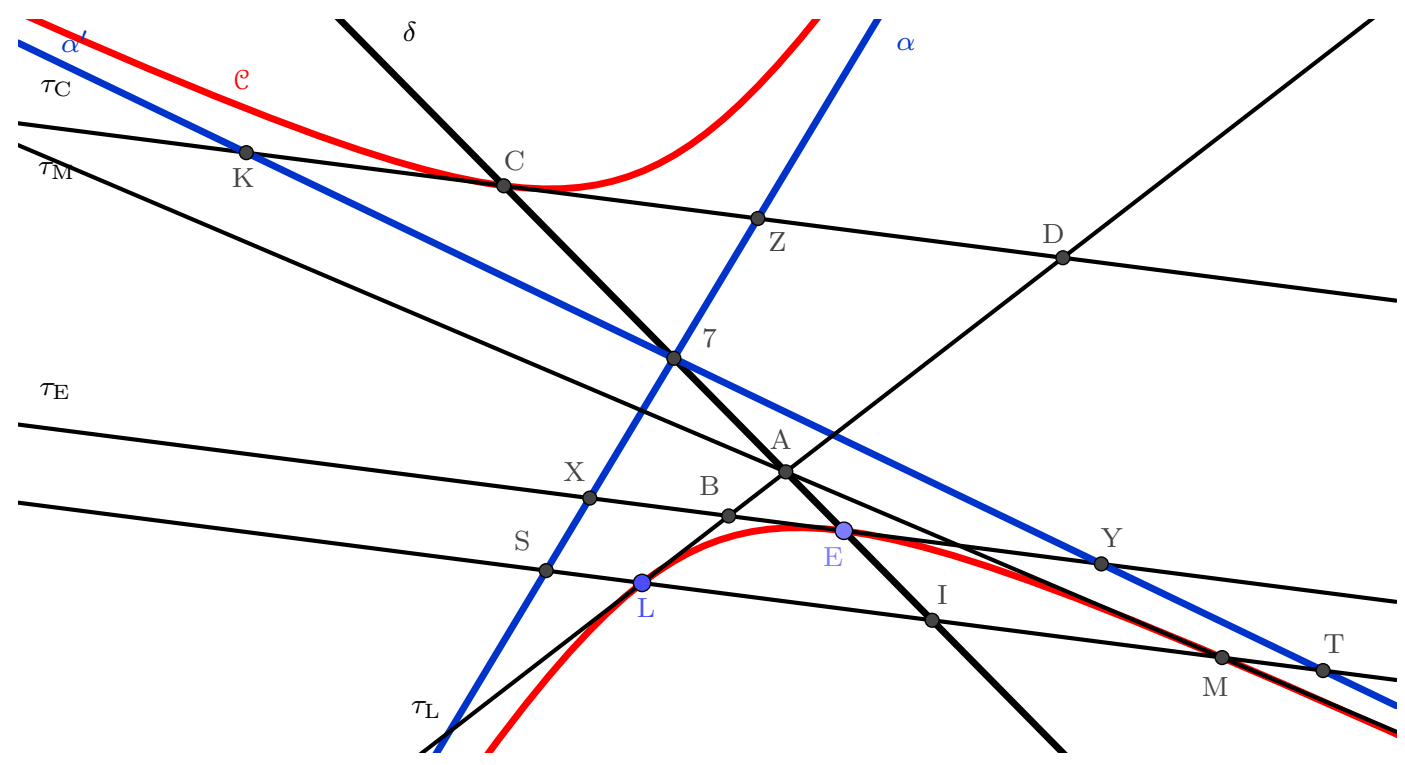

FIGURE s - L'hyperbole considérée par Desargues.

Comme nous l'avons noté, (LM) est la traversale de $\mathrm{A}$ eu égard à $\mathcal{C}$ et donc A, I; E, E; C, C forment une involution. Comme 7 est milieu du segment des nouds moyens doubles, il est accouplé au point à l'infini sur le tronc ou ordonnée $\delta$. 
Comme $\delta$ est un diamètre, on a IL $=$ IM et Desargues énonce ${ }^{31}$ qu'outre cette égalité, on a également IS $=\mathrm{IT}, \mathrm{LS}=\mathrm{MT}$ et $\mathrm{MS}=\mathrm{LT}$, ce qu'il déclare être évident $\ll$ au moyen d'une ramée ${ }^{32}$. » Voici comment on peut entendre cette affirmation. Notons $\Delta_{\infty}$ la droite « dont tous les points sont à distance infinie ${ }^{33} \gg$ et donnons des noms aux points à l'infini des droites suivantes : $\mathrm{S}_{\infty}$ celui de $\alpha, \mathrm{T}_{\infty}$ celui de $\alpha^{\prime}, \mathrm{I}_{\infty}$ celui de $\delta$ et $\mathrm{I}_{\infty}^{\prime}$ celui de (LM), voir la figure 6 où l'on a ramené la droite à l'infini à distance finie.

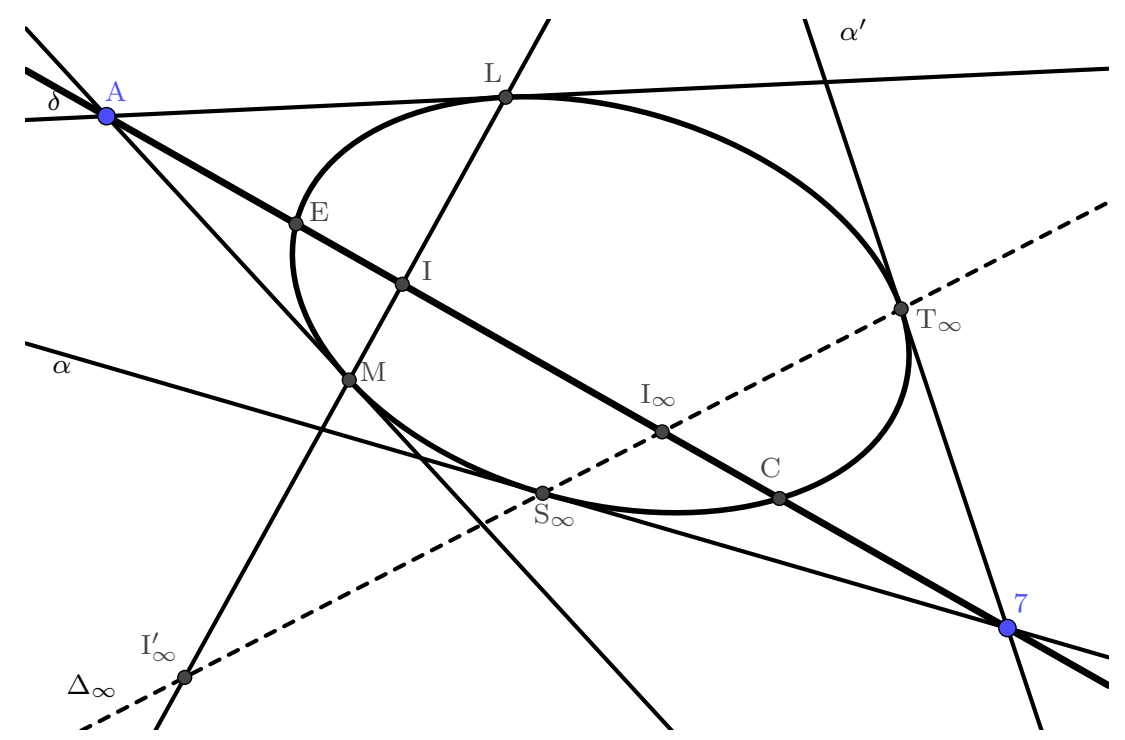

FIGURE 6 - Une ramée depuis la droite à l'infini.

D'après ce que Desargues écrit p. 23, lignes ı à i 5 , $\mathcal{C}$ rencontre $\Delta_{\infty}$ en les points $\mathrm{S}_{\infty}$ et $\mathrm{T}_{\infty}$ en lesquels elle admet pour tangentes les asymptotes $\alpha$ et $\alpha^{\prime}$. D'après le théorème d'involution appliqué à la droite à l'infini $\Delta_{\infty}$, les points $\mathrm{S}_{\infty}, \mathrm{S}_{\infty} ; \mathrm{T}_{\infty}, \mathrm{T}_{\infty} ; \mathrm{I}_{\infty}, \mathrm{I}_{\infty}^{\prime}$ forment une involution. Par ailleurs et comme $\delta=(\mathrm{EC})$ est un diamètre, les droites $\tau_{\mathrm{E}}, \tau_{\mathrm{C}}$ sont parallèles, tandis que (LM), ordonnée du même diamètre, leur est aussi parallèle. Ainsi $\tau_{\mathrm{E}}, \tau_{\mathrm{C}}$ et $(\mathrm{LM})$ sont parallèles, c'est-à-dire d'une même ordonnance dont le but est à l'infini en le point que nous avons noté $\mathrm{I}_{\infty}^{\prime}$. Par une ramée de sommet 7 de $\Delta_{\infty}$ vers la droite $(\mathrm{LM})$, il en découle que $\mathrm{I}, \mathrm{I}_{\infty}^{\prime} ; \mathrm{S}, \mathrm{S} ; \mathrm{T}, \mathrm{T}$ forment une involution et donc que I est milieu du segment $[\mathrm{ST}]$. Ainsi IS $=\mathrm{IT}$ et les deux autres égalités énoncées par Desargues en découlent immédiatement.

La phrase suivante ${ }^{34}$ poursuit dans cette veine purement projective et donc tout à fait révolutionnaire :

«Et ensuite, que le rectangle des pièces d'une diamétrale E7C, contenues entre son ordonnée des atouchements à la figure par ces asymptotes à distance infinie, \& chacun des points comme E, \& C, qu'y donne le bord de la figure est au rectangle des brins déployés de cette ordonnée ainsi à distance infinie contenus entre cette diamétrale E7C $\&$ les deux points qu'y donne le bord de la figure, en même raison que le rectangle comme IE, IC est au rectangle comme IL, IM » etc.

\footnotetext{
3i. p. 26,1 . IO-I 3

32. p. 26, Advertissements pour la ligne I 3 .

33. p. i6, Advertissements pour la ligne 3.

34. p. $26,1.14-22$
} 
Les atouchements en l'infini, ce sont les points $S_{\infty}, T_{\infty}$. Leur ordonnée, eu égard ici au diamètre choisi $\delta$, c'est donc la droite $\mathrm{S}_{\infty}, \mathrm{T}_{\infty}, \mathrm{c}$-à-d. la droite à l'infini $\Delta_{\infty}$. Le « rectangle des pièces d'une diamétrale $\mathrm{E} 7 \mathrm{C} \gg$ désigne donc le rectangle $\mathrm{I}_{\infty} \mathrm{E} . \mathrm{I}_{\infty} \mathrm{C}$. Les «brins déployés de cette ordonnée ainsi à distance infinie » sont alors les brins de la droite à l'infini $\mathrm{I}_{\infty} \mathrm{S}_{\infty}, \mathrm{I}_{\infty} \mathrm{T}_{\infty}$, qui sont bien déployés au tronc $\delta$ (noter ici l'analogie de situation avec celle décrite plus haut, à la figure 4). Desargues affirme donc dans les lignes citées ci-dessus que l'on a l'identité suivante :

$$
\frac{\mathrm{I}_{\infty} \mathrm{E} \cdot \mathrm{I}_{\infty} \mathrm{C}}{\mathrm{I}_{\infty} \mathrm{S}_{\infty} \cdot \mathrm{I}_{\infty} \mathrm{T}_{\infty}}=\frac{\mathrm{IE} \cdot \mathrm{IC}}{\mathrm{IL} \cdot \mathrm{IM}}
$$

En effet, si l'on note comme plus haut EF le paramètre de la conique eu égard au diamètre $\delta$, on a

$$
\frac{\mathrm{IE} . \mathrm{IC}}{\mathrm{IL} \cdot \mathrm{IM}}=\frac{\mathrm{EC}}{\mathrm{EF}},
$$

et le deuxième membre ne dépend pas du choix de L, ou plutôt de I, qui définit de manière univoque $L$ et $M$. Il suffit alors de remarquer que quand I tend vers $I_{\infty}$, alors $L$ tend vers $S_{\infty}$ et $M$ vers $T_{\infty}$, justifiant l'identité ci-dessus. En termes modernes, elle est conséquence de la continuité du birapport dans le plan projectif. Desargues poursuit en rappelant qu'on a également démontré que

$$
\frac{\mathrm{EC}}{\mathrm{EF}}=\frac{7 \mathrm{E} \cdot 7 \mathrm{C}}{\mathrm{EX} \cdot \mathrm{CZ}}
$$

Or, d'après le théorème de Thalès,

$$
\frac{\mathrm{E} 7}{\mathrm{EX}}=\frac{\mathrm{I} 7}{\mathrm{IS}}, \text { et donc } \frac{\mathrm{E} 7^{2}}{\mathrm{EX}^{2}}=\frac{\mathrm{I} 7^{2}}{\mathrm{IS}^{2}}
$$

et, comme $7 \mathrm{E}=7 \mathrm{C}$ et $\mathrm{EX}=\mathrm{CZ}$, on peut conclure que 35

$$
\frac{\mathrm{E} 7^{2}}{\mathrm{EX}^{2}}=\frac{\mathrm{I} 7^{2}}{\mathrm{IS}^{2}}=\frac{7 \mathrm{E} .7 \mathrm{C}}{\mathrm{EX} . \mathrm{CZ}}=\frac{\mathrm{IC} . \mathrm{IE}}{\mathrm{IL} . \mathrm{IM}}
$$

Il invoque alors ${ }^{36}$ les propositions 5 et 6 du livre II des Éléments d'Euclide pour dire que

$$
\mathrm{IE} . \mathrm{IC}+\mathrm{E} 7^{2}=\mathrm{I} 7^{2}
$$

et

$$
\mathrm{LS} . \mathrm{LT}+\mathrm{IM}^{2}=\mathrm{IS}^{2} .
$$

De l'identité

$$
\frac{\mathrm{I}^{2}}{\mathrm{IS}^{2}}=\frac{\mathrm{IC} \cdot \mathrm{IE}}{\mathrm{IL} \cdot \mathrm{IM}}=\frac{\mathrm{IE} \cdot \mathrm{IC}}{\mathrm{IM}^{2}}
$$

on peut alors tirer

$$
\frac{\mathrm{I}^{2}}{\mathrm{IS}^{2}}=\frac{\mathrm{I}^{2}-\mathrm{IE} \cdot \mathrm{IC}}{\mathrm{IS}^{2}-\mathrm{IM}^{2}}=\frac{7 \mathrm{E}^{2}}{\mathrm{LT} . \mathrm{SL}}
$$

35. p. $26,1.23-25$

36. p. $26,1.26$ 
Comme par ailleurs, d'après la théorie du paramètre,

$$
\frac{\mathrm{I}^{2}}{\mathrm{IS}^{2}}=\frac{\mathrm{IC} \cdot \mathrm{IE}}{\mathrm{IL} \cdot \mathrm{IM}}=\frac{\mathrm{EC}}{\mathrm{EF}}
$$

on peut conclure que LT.SL $=7 \mathrm{E}^{2}$. $(\mathrm{EF} / \mathrm{EC})$, et 7 étant milieu de $\mathrm{EC}$, nous obtenons finalement ${ }^{37}$ l'identité

$$
\text { LT.LS }=\frac{1}{4} \mathrm{EF} . \mathrm{EC},
$$

ce qui achève sa brève étude de l'hyperbole.

Desargues évoque ensuite les diverses possibilités pour deux cônes de se contenir ou de se toucher, précisant les diverses conclusions que l'on peut en tirer sur leurs sections coniques respectives. C'est sans aucun doute à rattacher au passage décrit dans la section sur les souches communes à plusieurs arbres, voir plus haut. Il propose ensuite, de manière vague mais juste, une extension de la théorie des traversales, ou de la polarité, aux massifs « qui ont rapport à la boule comme l'ellipse au cercle, » autrement dit aux quadriques. Comme il ne s'étend pas sur ce sujet, nous laissons cet aspect de côté et passons à l'analyse de la dernière partie du Brouillon Project contenant des propositions et leurs preuves.

\section{Perspective et foyers}

Nous allons maintenant examiner la dernière portion du Bronillon Project au contenu strictement mathématique. Desargues y aborde des questions qui sont à la fois dans la tradition apollinienne et dans la continuité de ses préoccupations pour la perspective. Ce développement est assez long, puisqu'il court de la ligne 8 de la page 27 à la ligne 6 de la page 29 et, comme souvent, on n'en comprend le but qu'à la toute fin. Ainisi la conséquence principale de ce long passage pourrait être énoncée de la façon suivante :

Théorème 6.I. - Soient $\mathcal{C}$ une conique d'un plan $\mathcal{P}, \delta$ une droite coupant la conique en deux points, et X un point de $\delta$ qui ne soit pas sur la conique. Alors il existe un point $\theta$ hors de $\mathcal{P}$ et un plan $\mathcal{Q}^{\prime}$ qui coupe le cône de base $\mathcal{C}$ et de sommet $\theta$ selon une conique $\mathrm{C}^{\prime}$ dont l'un des foyers soit l'image par perspective depuis le sommet $\theta d u$ point $\mathrm{X}$ et dont le grand axe soit l'image par perspective depuis le sommet $\theta$ de la droite $\delta$.

La démonstration de ce théorème passe par celle de quelques lemmes et est longue et technique. Nous allons en donner pour commencer un court résumé, afin de permettre au lecteur qui ne voudrait pas s'attarder sur les détails d'en avoir une idée assez précise.

\section{I Un bref résumé de la construction}

Donnons-nous $\mathrm{F}$ un autre point sur $\delta$ et à l'intérieur de la conique. Soit $\Delta$ la polaire de $\mathrm{F}$ eu égard à $\mathrm{C}$ qui, de la sorte, ne coupe pas la conique. La droite $\Delta$ coupe la droite $\delta$ en un point $\mathrm{A}$ et l'on choisit alors un point $\mathrm{Q}$ sur $\delta$ qui soit conjugué harmonique de

37. p. $26,1 \cdot 33-37$ 
X eu égard au couple A, F. Ainsi les couples A, F; X, X; Q, Q forment une involution dont $\mathrm{X}$ et $\mathrm{Q}$ sont des nœuds moyens doubles. Soit $\mathrm{P}$ le milieu du segment $[\mathrm{XQ}]$ et soit 7 le centre de la conique $\mathcal{C}$. La droite $(7 \mathrm{P})$ coupe la droite $\Delta$ en un point $\mathrm{C}$. Traçons par A l'une des tangentes à $\mathcal{C}$ et notons $\mathrm{H}$ le point de contact de cette tangente avec la conique; traçons la droite $(\mathrm{FH})$ et notons $\mathrm{D}$ son point d'intersection avec $\Delta$. Donnonsnous enfin un autre couple $\mathrm{R}, \mathrm{Z}$ de nœuds de l'involution ci-dessus, traçons par $\mathrm{Z}$ une tangente à $\mathcal{C}$, qui coupe $\Delta$ en un point $\mathrm{B}$. Du point de contact $\mathrm{G}$ de cette même tangente menons la droite GR qui va couper $\Delta$ en un point $E$.

Le résultat central de tout ce passage est que les points $\mathrm{A}, \mathrm{D} ; \mathrm{B}, \mathrm{E}$ sont des couples de nœuds d'un arbre de souche $\mathrm{C}$, soit donc que l'on a l'identité de rectangles $\mathrm{CA}$.CD = CE.CB. Remarquons au passage que si $\mathcal{C}, \mathrm{X}, \delta$ et $\mathrm{F}$ sont donnés de position, alors toute la construction peut se faire à la règle et au compas.

Passons maintenant à la construction perspectiviste et donnons-nous un plan $Q$ coupant le plan de la conique $e$ selon la droite $\Delta$. Traçons dans ce plan la droite $\Lambda$ passant par $\mathrm{C}$ et perpendiculaire à $\Delta$. L'arbre C; A, D; B, E donne naissance à une involution qui a des nœuds moyens; nommons W l'un d'entre eux. Dire que c'est un nœud moyen signifie que $\mathrm{CW}^{2}=\mathrm{CA} \cdot \mathrm{CD}=\mathrm{CE}$. $\mathrm{CB}$, soit encore que

$$
\frac{\mathrm{CA}}{\mathrm{CW}}=\frac{\mathrm{CW}}{\mathrm{CD}} \text {. }
$$

Ainsi CW est moyenne proportionnelle de CA et CD. Or la proposition i 3 du livre VI des Éléments d'Euclide permet de construire une telle moyenne proportionnelle : traçons dans $Q$ un demi-cercle de diamètre $\mathrm{AD}$ et nommons $\theta$ le point d'intersection de ce demi-cercle avec $\Lambda$ : C $\theta$ est la moyenne proportionnelle de CA et CD et $\theta$ est le sommet du cône de base $\mathcal{C}$ recherché pour finir la construction. Soit en effet $\mathcal{Q}^{\prime}$ un plan parallèle à $Q$ ne passant pas par $\theta$. Il va couper le cône de sommet $\theta$ et de base $\mathcal{C}$ selon une conique $\mathrm{C}^{\prime}$ qui est une vue en perspective de la conique $\mathrm{C}$. Les vues des points $\mathrm{X}$ et $\mathrm{Q}$ dans cette même perspective sont les points $\mathrm{X}^{\prime}, \mathrm{Q}^{\prime}$ intersections des droites $(\theta \mathrm{X})$ et $(\theta \mathrm{Q})$ avec le plan $Q^{\prime}$. Desargues affirme alors sans justification que ces deux points sont les foyers de la coniques $\mathrm{C}^{\prime}$, ce qui achève la démonstration du théorème. Nous verrons qu'en se rappelant certaines constructions par perspective données un peu avant dans le Brouillon, comme par exemple celle du centre ou de diamètres conjugués, il est relativement aisé de retrouver la preuve de ce remarquable résultat.

Nous reproduisons ${ }^{38}$, au sujet de cette très belle construction, la figure issue de la copie manuscrite du Brouillon Project faite par Philippe de la Hire et y portant le numéro i 9. Sur celle-ci, la conique $\mathcal{C}$ est représentée par le cercle en haut de la figure, et le demi-cercle du bas de la même figure est à comprendre comme tracé sur le plan incliné $\mathrm{Q}$. Le mieux alors pour bien comprendre la construction est de plier la figure le long de $\Delta$ et de regarder le dièdre ainsi obtenu depuis le dessus, en gardant face à soit le demi cercle, le cercle supérieur de la figure apparaissant alors comme une ellipse, la conique de départ $\mathcal{C}$. La droite que nous avons nommée $\Delta$ (resp. $\delta$ ) y est figurée par la droite (AV) (resp. (XA)).

Nous allons maintenant analyser en détail ce long passage menant à la démonstration du théorème 6.I.

38. voir la figure 7 . 


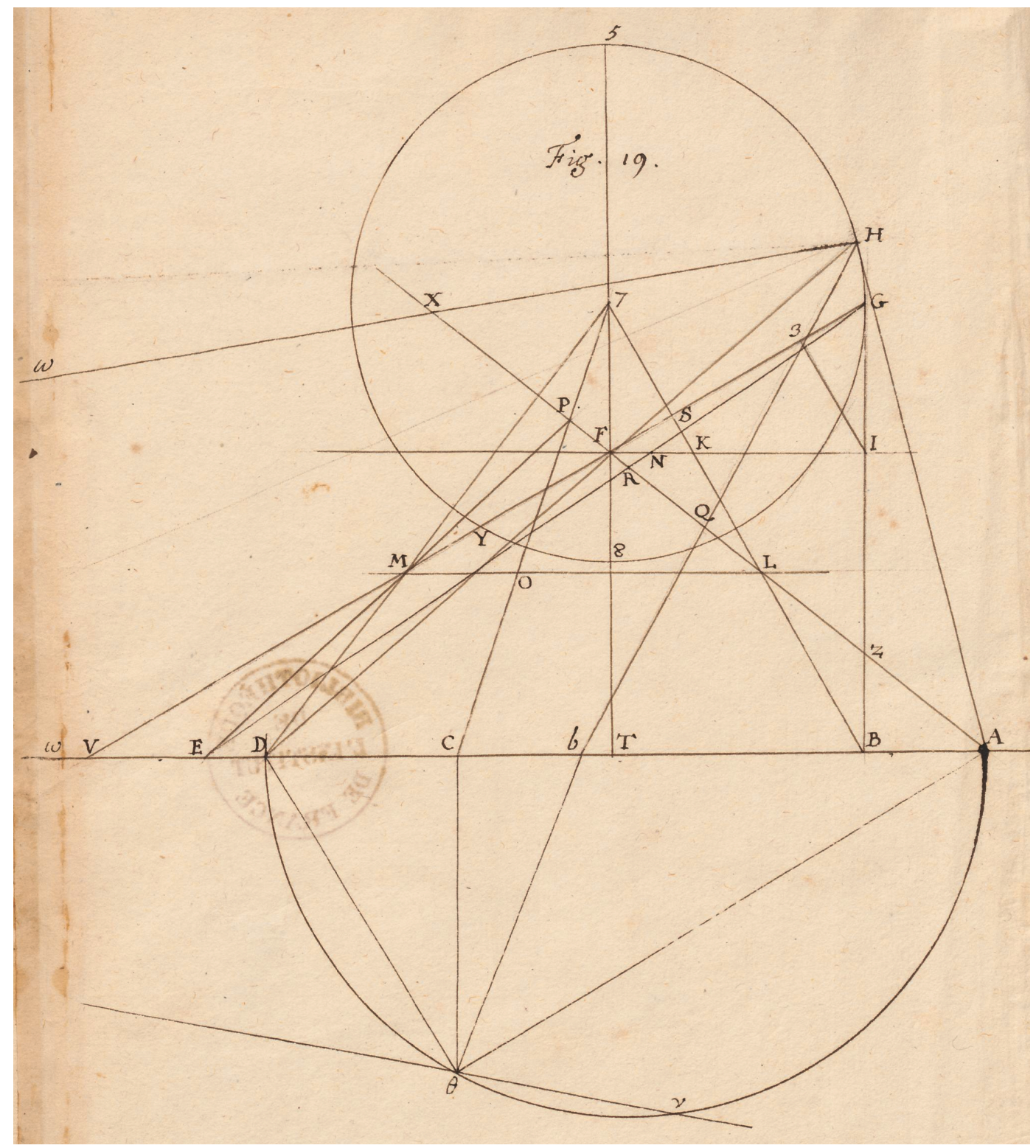

FIGURE 7 - La figure de la Hire illustrant la construction de Desargues.

\subsection{La construction d'un arbre dans le plan de la conique}

Soient $\mathcal{C}$ une conique, $\mathrm{F}$ un point pris hors de la conique et $\Delta$ la traversale de $\mathrm{F}$ eu égard à $\mathrm{C}$. Soit $\mathrm{A}$ un point sur $\Delta$, traçons la droite $\delta=(\mathrm{AF})$. Soient deux points $\mathrm{X}, \mathrm{Q}$ sur $\delta$ tels que A, F; X, X; Q, Q forment une involution ${ }^{39}$. Ainsi A, F; X, Q est une division harmonique. Soit 7 le centre de la conique. La droite $(7 \mathrm{~F})$ est donc un diamètre et l'une des ordonnées de la traversale $\Delta$. Son point traversal sera noté $T$ et ses points d'intersection avec la conique 5 et 8 . Soit $\mathrm{H}$ le point de contact d'une des tangentes à $\mathcal{C}$ passant par $\mathrm{A}$, tangente que nous noterons $\tau_{\mathrm{H}}$. Soit $\mathrm{D}$ le point traversal de l'ordonnée $(\mathrm{HF})$. Soit $\mathrm{P}$ le milieu du segment $[\mathrm{XQ}]$ et notons $\mathrm{C}$ le point de rencontre entre $(7 \mathrm{P})$

39. p. $27,1.8$-I I 
et $\Delta$. Soit $\mathrm{R}, \mathrm{Z}$ un autre couple de nœuds de l'involution $\mathrm{A}, \mathrm{F} ; \mathrm{X}, \mathrm{X} ; \mathrm{Q}, \mathrm{Q}$, de sorte que par Z on puisse tracer une tangente $\tau_{\mathrm{G}}$ à la conique. Notons B l'intersection de $\tau_{\mathrm{G}}$ avec $\Delta$, E celle de (GR) avec $\Delta$, et $\mathrm{V}$ le point traversal de l'ordonnée (GF).

Par construction, le point $\mathrm{P}$ est souche de l'arbre correspondant à l'involution A, F; R, Z; X, X; Q, Q et donc

$$
\text { PA.PF }=\text { PR.PZ }=\mathrm{PX}^{2}=\mathrm{PQ}^{2}
$$

De même, T, F; 5, 5; 8, 8 forment une involution et l'arbre correspondant est de souche 7 , milieu du segment [58]. Desargues écrit ${ }^{40}$ qu'il y a évidemment trois cas à traiter :

I. celui où $\mathrm{X}$ et $\mathrm{Q}$ sont sur la conique;

2. celui où ils lui sont tous deux intérieurs;

3. celui où ils lui sont tous deux extérieurs.

Il va brièvement étudier le premier cas puis traiter de manière unifiée les deux derniers. La première proposition s'énonce ainsi :

Proposition 6.I. - Si X et $\mathrm{Q}$ sont sur $\mathrm{C}$, alors $\mathrm{C}=\mathrm{D}$, dit autrement, $(7 \mathrm{P}),(\mathrm{HF})$ et $\Delta$ sont de même ordonnance.

En effet ${ }^{41}$, le point 7 est souche de l'arbre décrit ci-dessus donc il est accouplé au point à l'infini $\infty_{7 \mathrm{~F}}$ de la droite $(7 \mathrm{~F})$. De même, $\mathrm{P}$ est accouplé au point à l'infini $\infty_{\mathrm{PF}}$ de la droite $(\mathrm{PF})$. Enfin, la traversale de 7 est la droite à l'infini $\left(\infty_{7 \mathrm{~F}} \infty_{\mathrm{PF}}\right)$ et la traversale de $\mathrm{P}$ passe par $\infty_{\mathrm{PF}}$ qui est l'accouplé de P. Ainsi $(7 \mathrm{P})$ est traversale du point commun aux traversales de 7 et de $\mathrm{P}$, c'est-à-dire du point $\infty_{\mathrm{PF}}$. Ainsi $(7 \mathrm{P})$, (HF) et $\Delta$ sont traversales respectives des trois points alignés $\infty_{\mathrm{PF}}, \mathrm{A}$ et $\mathrm{F}$ et sont donc concourantes, ce qui démontre la proposition 6. I.

Il énonce alors une proposition dont la démonstration est similaire :

Proposition 6.2. - Sous la même hypothèse que $\mathrm{X}$ et $\mathrm{Q}$ sont sur $\mathrm{C}$, les points $\mathrm{C}$ et $\mathrm{E}$ sont confondus.

Revenons maintenant au cas général où ni $\mathrm{X}$ ni $\mathrm{Q}$ ne sont sur la conique. Un peu avant le passage traitant des deux cas particuliers ci-dessus ${ }^{42}$, il énonce le fait que E, B; D, A sont des couples de nœuds d'un arbre de souche C, ce qu'il ne démontrera que plus loin, mais qu'il réénonce ainsi ${ }^{43}$ :

Proposition 6.3. - Si X, Q ne sont pas sur la conique, alors CD.CA = CE.CB.

L'arbre est à souche engagée ou dégagée suivant que X, Q sont « de la part du concave » ou « de la part du convexe » de la conique ${ }^{44}$.

La démonstration de la proposition, «par le plan », découle de deux « circonstances $\gg$ auxquelles il est dit que «M. Pujos a très bonne part ${ }^{45} \gg$. Nous énoncerons ces deux « circonstances » sous la forme de deux lemmes.

\footnotetext{
40. p. 27. 1. 26-28

4I. p. $27,1.29-34$

42. p. 27,1 . I 7

43. p. $27,1.59-6 \mathrm{I}$

44. p. $27,1.4 \mathrm{I}-44$

45 . p. $27,1.54-55$
} 
Traçons la droite $(\mathrm{GF})$ et notons $\mathrm{Y}$ son deuxième point d'intersection avec $\mathcal{C}$. Notons $\mathrm{M}$ le point d'intersection de (7D) avec (GF) et $\mathrm{L}$ celui de (7B) avec (AF). Par $\mathrm{F}$, traçons la parallèle à $\Delta$, qui va couper (7B) en $\mathrm{K},(\mathrm{GE})$ en $\mathrm{N}$ et $(\mathrm{GB})$ en I. Par I, traçons la parallèle à $(7 \mathrm{~B})$, qui va couper $(\mathrm{GF})$ en 3 , et notons $\mathrm{O}$ le point de rencontre de (LM) et $(7 \mathrm{P})$.

Lemme 6.I. - La droite (LM) est parallèle à la droite $\Delta$.

Lemme 6.2. - Les droites (EP), (7D), (FV) et (LM) sont concourantes en $\mathrm{M}$.

La démonstration du premier lemme couvre les lignes i à 9 de la page 28. D'après le théorème de Ménélaüs, nous avons les identités suivantes :

$$
\frac{7 \mathrm{~T}}{7 \mathrm{~F}}=\frac{\mathrm{DT}}{\mathrm{DV}} \frac{\mathrm{MV}}{\mathrm{MF}} \text { et } \frac{7 \mathrm{~T}}{7 \mathrm{~F}}=\frac{\mathrm{BT}}{\mathrm{BA}} \frac{\mathrm{LA}}{\mathrm{LF}}
$$

d'où suit que ${ }^{46}$

$$
\frac{\mathrm{DT}}{\mathrm{DV}} \frac{\mathrm{MV}}{\mathrm{MF}}=\frac{\mathrm{BT}}{\mathrm{BA}} \frac{\mathrm{LA}}{\mathrm{LF}} .
$$

Cependant, la traversale de A est (HF), et (HF) coupe $\Delta$ en D. La traversale de B est (GF), et GF coupe $\Delta$ en $\mathrm{V}$. La traversale de 7 est la droite à l'infini, et (7F) coupe $\Delta$, traversale de $\mathrm{F}$, en $\mathrm{T}$, donc la traversale de $\mathrm{T}$ est la droite $\left(\mathrm{F} \infty_{\Delta}\right)$, c'est-à-dire la droite (FK) parallèle à $\Delta$. Ainsi $\mathrm{A}, \mathrm{D} ; \mathrm{B}, \mathrm{V} ; \mathrm{T} \infty_{\Delta}$ forment une involution et $\mathrm{T}$ est souche de l'arbre correspondant, donc TA.TD $=$ TB.TV et donc

$$
\frac{\mathrm{DT}}{\mathrm{TV}}=\frac{\mathrm{BT}}{\mathrm{TA}} .
$$

Mais DV (resp. BA) est somme ou différence de DT et TV (resp. de BT et TA) et ainsi ${ }^{47}$ :

$$
\frac{\mathrm{DT}}{\mathrm{DV}}=\frac{\mathrm{BT}}{\mathrm{BA}} .
$$

En simplifiant dans l'identité de rapports obtenue plus haut, on en conclut que

$$
\frac{\mathrm{MV}}{\mathrm{MF}}=\frac{\mathrm{LA}}{\mathrm{LF}}
$$

et donc, par la réciproque du théorème de Thalès, que (LM) est parallèle à $(\mathrm{AV})=\Delta$, ce qui démontre le premier lemme.

La démonstration du second lemme est plus longue et couvre les lignes io à 4I de la page 28. Par construction de la traversale, G, G; Y, Y; F, V forment une involution. Soit $\mathrm{S}$ la souche de l'arbre correspondant, c'est-à-dire le milieu du segment [GY]. Nous avons donc $\mathrm{SY}^{2}=\mathrm{SG}^{2}=\mathrm{SV}$.SF et, d'après une propriété de l'involution démontrée plus haut dans le Brouillon, aux lignes in et 20 de la page 5 , il s'ensuit que ${ }^{48}$

$$
\frac{\mathrm{GV}}{\mathrm{GF}}=\frac{\mathrm{SG}}{\mathrm{SF}} .
$$

46. p.28, 1. 6-7

47. p. $28,1.8$

48. p. $28,1.17$ 
Par construction, $(\mathrm{FN})$ est parallèle à $\Delta$ et $(\mathrm{B} 7)$ parallèle à $(\mathrm{I} 3)$ donc d'après le théorème de Thalès on $\mathrm{a}^{49}$ :

$$
\frac{\mathrm{GV}}{\mathrm{GF}}=\frac{\mathrm{VE}}{\mathrm{FN}}=\frac{\mathrm{GB}}{\mathrm{GI}}
$$

et

$$
\frac{\mathrm{GB}}{\mathrm{GI}}=\frac{\mathrm{GS}}{\mathrm{G} 3},
$$

donc

$$
\frac{\mathrm{GV}}{\mathrm{GF}}=\frac{\mathrm{GS}}{\mathrm{G} 3} .
$$

Nous pouvons donc déduire de 3 et 5 que

$$
\frac{\mathrm{GV}}{\mathrm{GF}}=\frac{\mathrm{SG}}{\mathrm{SF}}=\frac{\mathrm{SG}}{\mathrm{G} 3}
$$

et ainsi G3 = SF. Par somme ou différence, on en déduit aussi que F3 $=$ GS so.

Nous pouvons donc affirmer que

$$
\frac{\mathrm{F} 3}{\mathrm{FS}}=\frac{\mathrm{GV}}{\mathrm{GF}} .
$$

Cependant, du fait que la droite (SK), c'est-à-dire la droite (B7), est parallèle à la droite (3I), on déduit du théorème de Thalès que

$$
\frac{\mathrm{F} 3}{\mathrm{FS}}=\frac{\mathrm{FI}}{\mathrm{FK}} .
$$

Or, d'après 4 , on a

$$
\frac{\mathrm{GV}}{\mathrm{GF}}=\frac{\mathrm{VE}}{\mathrm{FN}},
$$

donc

$$
\frac{\mathrm{FI}}{\mathrm{FK}}=\frac{\mathrm{VE}}{\mathrm{FN}},
$$

soit finalement ${ }^{s I}$ VE.FK $=$ FI.FN.

Par construction $\mathrm{P}$ est souche de l'arbre associé à l'involution A, F; Z, R; X, X; Q, Q et donc, d'après une propriété générale des arbres ${ }^{52}$, on a

$$
\frac{\mathrm{PA}}{\mathrm{PF}}=\frac{\mathrm{AR} \cdot \mathrm{AZ}}{\mathrm{FR} \cdot \mathrm{FZ}}=\frac{\mathrm{RA}}{\mathrm{RF}} \frac{\mathrm{ZA}}{\mathrm{ZF}}
$$

Or, d'après le théorème de Thalès, nous avons

$$
\frac{\mathrm{ZA}}{\mathrm{ZF}}=\frac{\mathrm{AB}}{\mathrm{FI}} \text { et } \frac{\mathrm{RA}}{\mathrm{RF}}=\frac{\mathrm{EA}}{\mathrm{FN}}
$$

49. p. $28,1$. I 8 -I 9

5o. p. $28,1.20$

5I. p. $28,1.23-24$

52. p. $4,1.15-18$ 
donc

$$
\frac{\mathrm{PA}}{\mathrm{PF}}=\frac{\mathrm{EA}}{\mathrm{FN}} \frac{\mathrm{AB}}{\mathrm{FI}}=\frac{\mathrm{AE} \cdot \mathrm{AB}}{\mathrm{FN} \cdot \mathrm{FI}} .
$$

Comme FN.FI = VE.FK, il s'ensuit que

$$
\frac{\mathrm{PA}}{\mathrm{PF}}=\frac{\mathrm{AE} \cdot \mathrm{AB}}{\mathrm{VE} \cdot \mathrm{FK}}=\frac{\mathrm{EA}}{\mathrm{EV}} \frac{\mathrm{AB}}{\mathrm{FK}} .
$$

D'après le théorème de Thalès, on a $\mathrm{AB} / \mathrm{FK}=\mathrm{LA} / \mathrm{LF}$ d'où

$$
\frac{\mathrm{PA}}{\mathrm{PF}}=\frac{\mathrm{EA}}{\mathrm{EV}} \frac{\mathrm{LA}}{\mathrm{LF}} .
$$

Or, dans le cours de la démonstration du lemme 6.I, Desargues a démontré que $\mathrm{LA} / \mathrm{LF}=\mathrm{MV} / \mathrm{MF}$ et l'on peut finalement conclure que

$$
\frac{\mathrm{PA}}{\mathrm{PF}}=\frac{\mathrm{EA}}{\mathrm{EV}} \frac{\mathrm{MV}}{\mathrm{MF}} .
$$

Par « la converse d'une cy-dessus, » c'est-à-dire par la réciproque du théorème de Ménélaüs, nous pouvons en déduire ${ }^{\$ 3}$ que $\mathrm{P}, \mathrm{M}$ et $\mathrm{E}$ sont alignés, c'est-à-dire que $\mathrm{M}$ est sur la droite $(\mathrm{PE})$.

Par ailleurs et par construction, $\mathrm{M}$ est sur (7D), tandis que (SV) $=(\mathrm{GF})$ et que $\mathrm{M}$ est le point de concours de (7D) et (GF). Ainsi (LM), (7D) et (SV) sont concourantes en M. De ce qui précède découle alors que (EP), (7D), (FV) et (LM) sont concourantes en $\mathrm{M}$, ce qui achève la démonstration du lemme $6.2^{54}$.

Desargues peut alors démontrer la proposition 6.3 aux lignes 42 à 47 de la page 28. D'après le théorème de Thalès, que l'on est en droit d'appliquer grâce aux lemmes démontrés ci-desus,

$$
\frac{\mathrm{OL}}{\mathrm{OM}}=\frac{\mathrm{CA}}{\mathrm{CE}} \text { et } \frac{\mathrm{OL}}{\mathrm{OM}}=\frac{\mathrm{CB}}{\mathrm{CD}},
$$

et donc bien évidemment CA.CD $=$ CE.CB, ce qui termine la démonstration de la proposition 6.3 affirmant que A, D; E, B sont couples de nouds d'un arbre de souche C.

Desargues énonce ensuite "s, sans démonstration, que, selon les cas, l'arbre obtenu est à souche engagée (l'involution associée est elliptique) quand $\mathrm{X}$ et $\mathrm{Q}$ sont à l'intérieur de la conique, à souche dégagée (l'involution est hyperbolique) quand $\mathrm{X}$ et $\mathrm{Q}$ sont à l'extérieur de la conique, et « de l'espèce mitoyenne » (il n'y a pas d'involution dans ce cas) quand $\mathrm{X}$ et $\mathrm{Q}$ sont sur la conique.

\subsection{La construction d'une perspective}

Desargues achève cette partie du Bromillon Project par une application de ce qu'il vient de démontrer à une construction de nature perspectiviste dont nous avons résumé les éléments dans le théorème 6.I. Il n'en donne pas la justification, mais on peut juger,

53. p. $28,1.37$

54. Et le lecteur par la même occasion...

55. p. $28,1.48-52$ 
au vu de la figure ig du manuscrit de la Hire reproduite plus haut, que celui-ci en a retrouvé la teneur. Dans son édition allemande du Brouillon Project, Zacharias en a donné une reconstruction incomplète et fausse qui passe complètement à côté de l'aspect profondément perspectiviste de la construction du Lyonnais. Notons à ce sujet que René Taton a repris plusieurs des notes explicatives de Zacharias, parfois sans les citer, et que ce cas ci ne fait pas exception.

Rappelons les données de ce théorème : soient $\mathcal{C}$ une conique en un plan $\mathcal{P}, \delta$ une droite coupant $\mathcal{C}$ et $\mathrm{X}, \mathrm{F}$ deux points sur $\delta$ hors de la conique. Il s'agit de construire un point $\theta$ et un plan $Q^{\prime}$ de sorte que, dans une perspective de sommet $\theta$ de $\mathcal{P}$ vers $\mathcal{Q}^{\prime}$, la conique $\mathcal{C}$ (resp. la droite $\delta$ et le point $\mathrm{X}$ ) soit représentée par une conique $\mathcal{C}^{\prime}$ (resp. par une droite $\delta^{\prime}$ et un point $\mathrm{X}^{\prime}$ ) de manière à ce que $\delta^{\prime}$ soit le grand axe de $\mathrm{C}^{\prime}$ et $\mathrm{X}^{\prime}$ l'un de ses foyers. Dans la suite, nous utiliserons systématiquement des lettres « primées » pour désigner les représentations dans le plan $Q^{\prime}$ des objets de $\mathcal{P}$ désignés par la même lettre.

On peut, pour commencer, utiliser une construction perspectiviste déjà donnée par Desargues comme une première application de la théorie de la traversale, à la fin de la page 22 et au début de la page $23^{56}$ du Bronillon. Soient $\Delta$ la traversale de F eu égard à $\mathcal{C}$ et $Q$ un plan coupant $\mathcal{P}$ selon $\Delta$. Soit par ailleurs $Q^{\prime}$ un plan parallèle à $Q$. Alors, quelque soit le point $\theta$ de $\mathcal{Q}$ pris hors de $\Delta$, dans la perspective décrite ci-dessus, la droite $\Delta$ est envoyée sur la droite à l'infini du plan $Q^{\prime}$. Mais les perspectives envoient les traversales sur les traversales et ainsi la traversale de $\mathrm{F}^{\prime}$ eu égard à $\mathrm{C}^{\prime}$ est la droite à l'infini : le point $\mathrm{F}^{\prime}$ est donc le centre de $\mathrm{C}^{\prime}$ et $\delta^{\prime}$, passant par $\mathrm{F}^{\prime}$, en est un des diamètres.

Soit maintenant A le point d'intersection des droites $\delta$ et $\Delta$ et menons par A l'une des deux tangentes à $\mathcal{C}$, de sorte que cette droite soit la tangente $\tau_{\mathrm{H}}$ à $\mathcal{C}$ en un point $\mathrm{H}$. Elle est représentée dans $Q^{\prime}$ par la tangente $\tau_{\mathrm{H}}^{\prime}$ au point $\mathrm{H}^{\prime}$ et cette dernière, passant par le même point à l'inifini $\mathrm{A}^{\prime}$ que $\delta^{\prime}$, lui est parallèle : les deux diamètres $\delta^{\prime}$ et $\left(\mathrm{H}^{\prime} \mathrm{F}^{\prime}\right)$ de $\mathrm{C}^{\prime}$ sont conjugués. Il reste maintenant à choisir judicieusement le point $\theta$ dans $Q^{\prime}$ pour que ces deux diamètres soient perpendiculaires et soient donc les axes principaux de $\mathrm{C}^{\prime}$, et pour que $\mathrm{X}^{\prime}$ soit un foyer.

Soit $\mathrm{Q}$ le conjugué harmonique du point $\mathrm{X}$ par rapport à la paire $\mathrm{F}, \mathrm{A}$. De la sorte $\mathrm{F}, \mathrm{A} ; \mathrm{X}, \mathrm{X}, \mathrm{Q}, \mathrm{Q}$ est une involution de quatre points. Nous disposons ainsi de quatre droites (HF), (HA), (HX), (HQ) formant un pinceau harmonique, ou des droites harmonicales pour reprendre la terminologie de la Hire. Ces droites coupent $\Delta$ en les points $\mathrm{D}, \mathrm{A}, \omega$ et $b$ (ces deux derniers sont nommés par la Hire sur la figure 7) qui forment donc, d'après le théorème de la ramée, une involution de quatre points $\mathrm{D}, \mathrm{A} ; \omega, \omega ; b, b$. $\mathrm{Si} 7$ est le centre de $\mathcal{C}$ et $\mathrm{P}$ le milieu du segment [XQ], la droite (7P) coupe $\Delta$ au point $\mathrm{C}$ dont Desargues a démontré juste avant qu'il était souche d'un arbre dont $\mathrm{D}, \mathrm{A}$ sont un couple de nœuds. Cet arbre admet un nœud moyen $\mathrm{W}$ de sorte que $\mathrm{CA}$. $\mathrm{CD}=\mathrm{CW}^{2}$, ou encore

$$
\frac{\mathrm{CA}}{\mathrm{CW}}=\frac{\mathrm{CW}}{\mathrm{CD}} \text {. }
$$

Ainsi CW est moyenne proportionnelle des deux grandeurs CA, CD. C'est ce qui va nous permettre de trouver le sommet $\theta$ recherché.

Soit $\Lambda$ la droite de $\mathcal{Q}$ perpendiculaire à $\Delta$ en $\mathrm{C}$ et plaçons sur $\Lambda$ un point $\theta$ tel que

56. Voir aussi l'article [3]. 
$\mathrm{C} \theta=\mathrm{CW}$. Comme le montre la Hire dans sa figure, on peut, d'après la proposition $\mathrm{I} 3$ du livre VI des Éléments, obtenir ce point comme intersection du cercle de $Q$ de diamètre $[\mathrm{DA}]$ avec la droite $\Lambda$. En particulier, les droites $(\theta \mathrm{A})$ et $(\theta \mathrm{D})$ sont perpendiculaires.

Si parler de représentation en perspective de sommet $\theta$ de $\mathcal{P}$ vers le plan 2 , qui contient $\theta$, n'a pas de sens en ce qui concerne les points, cela en a un, par simple incidence de plans, en ce qui concerne les droites. En raisonnant sur les représentations des droites du faisceau harmonique sus-cité, on peut alors transiter par le plan $Q$, y exploiter les propriétés élémentaires d'un faisceau harmonique dont deux droites sont perpendiculaires, et aller ensuite dans le plan $Q^{\prime}$ où ces propriétés restent vraies du fait de son parallèlisme avec $Q$.

Considérons le pinceau de plans $(\theta \mathrm{HF}),(\theta \mathrm{HA}),(\theta \mathrm{HQ}),(\theta \mathrm{HX})$ d'essieu $(\theta \mathrm{H})$. Ces plans coupent $Q$ selon les droites respectives $(\theta \mathrm{D}),(\theta \mathrm{A}),(\theta \omega)$ et $(\theta b)$, qui forment un faisceau harmonique dont deux droites $(\theta \mathrm{D}),(\theta \mathrm{A})$ sont perpendiculaires : elles sont donc les bissectrices des deux autres droites $(\theta \omega),(\theta b)$.

Passons maintenant au plan $Q^{\prime}$, parallèle au plan $Q$ et reprenons la convention sur les lettres primées mentionnée plus haut. Les points $\mathrm{A}^{\prime}, \mathrm{F}^{\prime} ; \mathrm{X}^{\prime}, \mathrm{X}^{\prime} ; \mathrm{Q}^{\prime}, \mathrm{Q}^{\prime}$ forment une involution de quatre points. L'accouplé $\mathrm{A}^{\prime}$ de $\mathrm{F}^{\prime}$ est à distance infinie puisque sur $\Delta^{\prime}$ et donc $\mathrm{F}^{\prime}$ est le milieu du segment $\left[\mathrm{X}^{\prime} \mathrm{Q}^{\prime}\right]$. La droite $\left(\mathrm{H}^{\prime} \mathrm{F}^{\prime}\right)$, représentée par $(\theta \mathrm{D})$ dans $Q$, est perpendiculaire à $\delta^{\prime}$, représentée par $(\theta \mathrm{A})$ dans $Q$. Ainsi les deux diamètres conjugués $\delta^{\prime}$ et $\left(\mathrm{H}^{\prime} \mathrm{F}^{\prime}\right)$ sont-ils perpendiculaires et sont donc les axes principanx de la conique $\mathrm{C}^{\prime}$.

La tangente à $\mathcal{C}^{\prime}$ en $\mathrm{H}^{\prime}$ est représentée dans $\mathcal{Q}$ par la droite $(\theta \mathrm{A})$ (c'est la même que celle représentant $\left(\mathrm{H}^{\prime} \mathrm{F}^{\prime}\right):\left(\mathrm{H}^{\prime} \mathrm{F}^{\prime}\right)$ est parallèle à $\left.\tau_{\mathrm{H}}^{\prime}\right)$. Les droites $\left(\mathrm{H}^{\prime} \mathrm{X}^{\prime}\right)$ et $\left(\mathrm{H}^{\prime} \mathrm{Q}^{\prime}\right)$ y sont représentées respectivement par les droites $(\theta \omega)$ et $(\theta b)$. Or $(\theta \mathrm{A})$ est bissectrice des droites $(\theta \omega),(\theta b)$ et le plan $Q^{\prime}$ est parallèle au plan $Q$, donc $\left(\mathrm{H}^{\prime} \mathrm{X}^{\prime}\right)$ est bissectrice des droites $\left(\mathrm{H}^{\prime} \mathrm{Q}^{\prime}\right),\left(\mathrm{H}^{\prime} \mathrm{X}^{\prime}\right)$ : les deux points $\mathrm{X}^{\prime}, \mathrm{Q}^{\prime}$ sont les foyers de $\mathrm{C}^{\prime}$, ce qui achève la justification de cette très élégante construction d'inspiration perspectiviste de Desargues.

\section{Conclusion}

Comme le montrent les pages qui précèdent, on ne peut avoir de doute sur le fait que Girard Desargues, dans son Brouillon Project sur les coniques, raisonne de manière résolument projective. Son utilisation pleine et entière des éléments à l'infini, traités exactement comme ceux se situant à distance finie, donnent raison à Jean-Victor Poncelet qui le qualifiait de père de la géométrie projective. Des questions demeurent cependant en suspens. Ainsi, il ne semble pas que Desargues, ni son élève direct Blaise Pascal, ait pris la mesure de l'ampleur de la nouveauté que représentait cette manière de faire rompant avec les pratiques et conceptions de la géométrie euclidienne. Pourquoi Desargues limite-t-il la portée de son innovation au seul traitement des coniques et ne s'en saisit-il pas pour fonder une nouvelle géométrie? Outre le fait que l'émergence de nouveaux concepts prend beaucoup de temps, on peut, nous semble-t-il, y voir la marque des motivations pratiques derrière les travaux géométriques du Lyonnais.

La lecture du Bronillon Project et des quelques autres textes mathématiques attribuables à Girard Desargues montre bien que chez lui l'inspiration provient de la pratique de la perspective. Il est utile ici de citer la toute fin du petit traité de perspective de 1636 : 
« La proposition qui suit ne se dévide pas si brièvement que celles qui précèdent : ayant à pourtraire une coupe de cône plate, y mener deux lignes, dont les apparences soient les essieux de la figure qui la représentera. »

Précisons ce que Desargues affirme être en mesure de faire : soit une conique, située dans un certain plan et que l'on souhaite représenter en perspective dans un autre plan. Le résultat cette représentation est encore une conique. On peut construire, dans le plan de la conique que l'on représente, deux droites dont les apparences 57 soient les axes principaux de la représentation de cette conique.

La théorie projective des coniques développée par Girard Desargues dans le Brouillon Project, sous la forme entre autre de la notion de traversale, lui permet de remplir la sorte de programme qu'il s'est fixé en i636. Cela ressort bien des quelques lignes ${ }^{58}$ qui concluent le passage analysé dans la section précédente du présent article. Leur interprétation est suffisamment évidente au vu de ce qui précède pour que nous puissions les citer intégralement :

«De façon qu'étant pour assiette d'un cône, donnée de position une quelconque coupe de rouleau à bord courbe, \& en son plan une droite pour traversale comme AV, $\&$ l'angle du plan de cette coupe avec le plan qu'y donne le plan du sommet \& de cette traversale, \& en elle, ou bien la souche de l'arbre de cette construction comme ici le point $\mathrm{C}$, ou bien deux couples des nouds de cet arbre, ou bien hors d'elle un point tel que $\mathrm{P}$, ou bien un des points tels que $\mathrm{X}, \& \mathrm{Q}$, ou bien deux des couples de nouds de l'arbre comme XQ.

Le sommet de ce cône est donné de position, \& le cône est donné d'espèce \& de position, la figure de coupe qu'y donne cette position de plan de coupe est donnée d'espèce $\&$ de position, tous les diamètres conjugués de la figure de coupe avec leurs distinctions, toutes les ordonnées $\&$ touchantes avec leurs distinctions, les côtés coadjuteurs ${ }^{` 9}$, le but de l'ordonnance de ses diamétrales, \& les points foyers, y sont donnés chacun de génération, d'espèce, $\&$ de position.

Que si le sommet, l'assiette, la traversale, \& le plan de coupe sont donnés de position, tout le reste est donné semblablement de génération, d'espèce $\&$ de position. »

Le propos est donc entièrement centré sur les constructions géométriques autour des sections coniques, et il faut lire un peu plus avant pour trouver dans le texte quelques réflexions plus générales sur la géométrie. Dans une des dernières notes des Advertissements, Desargues fait une déclaration de sentiment concernant certaines de ses conceptions touchant la géométrie. Le problème sur lequel il bute est bien évidemment celui d'infini, qui pourra être contourné plus tard avec l'utilisation de coordonnées homogènes. Mais il écrit ceci : «En Géométrie on ne raisonne point des quantités avec cette distinction qu'elles existent ou bien effectivement en acte, ou bien seulement en puissance, ni du général de la nature, avec cette décision qu'il n'y ait rien en elle que l'entendement ne comprenne ${ }^{60}$. $\gg$ Il continue en montrant comment la droite, par exemple, ne peut être qu'infinie, puisqu'il n'y a quelqu'endroit où «l'imagination [ne] peut aller

57. ou représentations

58. p. 29, 1. I-I 3

59. c'est-à-direles paramètres.

6o. Advertissements, pour la ligne 3 de la page 30. La ponctuation a été modifiée pour la rendre conforme aux usages contemporains. 
en temps. Or jamais l'imagination ne peut aller en aucun endroit de l'espace auquel cet espace cesse de continuer; Donc l'espace \& conséquemment la droicte continüent toujours. » Il poursuit en étendant la validité de ces remarques aux quantités « si petites que leurs deux extremitez opposées sont unies entre elles » et conclut de manière prudente que, même si l'on « se sent incapable de comprendre l'une \& l'autre de ces deux espèces de quantités ${ }^{6 \mathrm{I}} \gg$ il ne faut pas en conclure qu'elles ne sont point en la nature, et que cette prudence doit s'étendre aux « propriétés qu'il a sujet de conclure de chacune d'elles, encore qu'elles semblent impliquer, à cause qu'il ne sauroit comprendre comment elles sont, telles qu'il les conclut par ses raisonnements. » Nous découvrons ici des idées qui ont clairement inspiré Blaise Pascal et qui seront développées presqu'en les même termes dans De l'esprit géométrique.

Mais les manières projectives de Desargues peuvent mener plus loin encore dans la révision de l'objet même de la géométrie. En introduisant la notion d'involution et celle qui lui est liée de traversale, notion invariante par perspective, Desargues prend conscience qu'il détient là un outil lui permettant d'énoncer des résultats qui sont valables quelque soit le point de vne que l'on adopte sur la figure considérée. Cela est mis à profit dans la dernière partie du Brouillon Project, analysée ci-dessus, où il utilise cette invariance pour construire un point de vue avantageux sur la figure. La figure n'est donc plus l'objet central de la géométrie. Blaise Pascal sera le premier à formuler ce changement fondamental en déclarant que la géométrie est la science de l'espace ${ }^{62}$. Nous espèrons, avec ce quatrième et dernier article d'analyse en profondeur du Brouillon Project de Girard Desargues, permettre un renouveau des réflexions sur la place et l'influence du Lyonnais dans l'histoire des mathématiques et, plus généralement, dans l'histoire des idées.

Remerciements. Les auteurs tiennent à remercier celles et ceux qui les ont soutenus lors de cette longue marche à travers le Browillon Project, plus particulièrement Philippe Abgrall, Sarah Ploquin-Donzenac et Sylvie Pic. Enfin, nous remercions le professeur Bernard Cache de l'École polytechnique fédérale de Lausanne pour sa lecture attentive du texte et ses illustrations interactives réalisées avec le logiciel TopSolid, qui nous ont permis de grandement améliorer le présent texte.

\section{Références}

[I] Marie Anglade and Jean-Yves Briend. La notion d'involution dans le Brouillon Project de Girard Desargues. Arch. Hist. Exact Sci., 7 I (6) :543-588, 2017.

[2] Marie Anglade and Jean-Yves Briend. L'usage de la combinatoire chez Girard Desargues : le cas du théorème de Ménélaüs. working paper or preprint hal-oi 692285, January 2018.

[3] Marie Anglade and Jean-Yves Briend. Le diamètre et la traversale : dans l'atelier de Girard Desargues. Archive for History of Exact Sciences, 73(4):385-426, 2019.

6r. les infiniment grandes et les infiniment petites.

62. Voir le fragment de l'Introduction à la Géométrie dans [13], p. 291-294. 
[4] Daniel Arasse. L'annonciation italienne : un histoire de perspective. Les Incontournables. Fernand Hazan, I 999, rééd. 2020.

[5] Jean-Yves Briend. Mathématiques en perspective : Desargues, la Hire, le Poîvre. working paper or preprint hal-02925995, August 2020.

[6] Girard Desargues and Max Zacharias. Erster Entwurf eines Versuchs über die Ergebnisse des Zusammentreffens eines Kegels mit einer Ebene, volume 197 of Ostwalds Klassiker der exakten Wissenschaften. Akademische Verlagsgesellschaft, Leipzig, I 922.

[7] Girard Desargues and Fabio Zanin. Abbozzo di un progetto d'indagine sulle conseguenze delle intersezioni del cono con un piano (1639), volume I I of Subsidia Mediaevalia Patavina. Il Poligrafo, 2006.

[8] Gustaf Eneström. Die "leçons de ténèbres " des Desargues. Bibl. Mathem., 3 :4 I I, 1902.

[9] Judith Field and Jeremy Gray. The geometrical work of Girard Desargues. SpringerVerlag, New York, I987.

[ı] Gilles-Gaston Granger. Essai d'une philosophie du style. Odile Jacob, Paris, I 988.

[ I I] Gottfried-Wilhelm Leibniz. Der Briefwechsel von G. W. Leibniz mit Mathematikern, herausgegeben von C. I. Gerhardt. Mayer \& Müller, Berlin, I 899.

[ 1 2] Claude Mydorge. Prodromi catoptricorum et dioptricorum : sive Conicorum operis ad abdita radii reflexi et refracti mysteria praevij et facem praeferentis, Libri quatuor priores. Petri Guillemot, Paris, I650.

[13] Blaise Pascal. Euvres de Blaise Pascal, publiées suivant l'ordre chronologique par Léon Brunschvicg, Pierre Boutroux et Félix Gazier. Librairie Hachette, Paris, I 9I4.

[ I4] René Taton. L'ouvre mathématique de G. Desargues. Bibl. de philosophie contemporaine Félix Alcan. Presses universitaires de France, Paris, I95 I.

[ I s] Max Zacharias. Desargues' Bedeutung für die projektive Geometrie. Deutsche Math., 5:446-457, I94I. 
Marie Anglade

Université d'Aix-Marseille

Centre Gilles Gaston Granger UMR CNRS 7304

3, place victor Hugo

I 333 I Marseille cede 3

France.

marie.anglade@univ-amu.fr

Jean-Yves Briend

Université d'Aix-Marseille

I2M UMR CNRS 7373

CMI

39, rue Joliot-Curie

I 3453 Marseille cedex I 3

France.

jean-yves.briend@univ-amu.fr 